\title{
Article
}

\section{Total Synthesis and Structural Reassignment of Laingolide A}

\author{
Fusong $W u^{1}$, Tao Zhang ${ }^{1}$, Jie $Y u^{1,2}$, Yian Guo ${ }^{1,2, * \mathbb{D}}$ and Tao Ye ${ }^{1, *}$ \\ 1 State Key Laboratory of Chemical Oncogenomics, Peking University Shenzhen Graduate School, \\ Shenzhen 518055, China; wufusong@pku.edu.cn (F.W.); 1901212908@pku.edu.cn (T.Z.); \\ yujie0701@pku.edu.cn (J.Y.) \\ 2 School of Biotechnology and Health Sciences, Wuyi University, Jiangmen 529020, China \\ * Correspondence: yianguo@pku.edu.cn (Y.G.); yet@pkusz.edu.cn (T.Y.)
}

Citation: Wu, F.; Zhang, T.; Yu, J.; Guo, Y.; Ye, T. Total Synthesis and Structural Reassignment of Laingolide A. Mar. Drugs 2021, 19, 247. https://doi.org/ 10.3390/md19050247

Academic Editor: Haruhiko Fuwa

Received: 7 April 2021

Accepted: 24 April 2021

Published: 27 April 2021

Publisher's Note: MDPI stays neutral with regard to jurisdictional claims in published maps and institutional affiliations.

Copyright: (C) 2021 by the authors. Licensee MDPI, Basel, Switzerland. This article is an open access article distributed under the terms and conditions of the Creative Commons Attribution (CC BY) license (https:// creativecommons.org/licenses/by/ $4.0 /)$.

\begin{abstract}
The asymmetric total synthesis of four diastereomers of laingolide A was achieved, which led to the unambiguous assignment of the stereochemistry of the natural product. The salient features of the convergent, fully stereocontrolled approach were a copper-catalysed stereospecific Kumadatype coupling, a Julia-Kocienski olefination and an RCM/alkene migration sequence to access the desired macrocyclic enamide.
\end{abstract}

Keywords: total synthesis; structural reassignment; laingolide A

\section{Introduction}

The modern structure determination of unknown natural products remains a challenging problem, especially when a small quantity of the natural compound is available, limiting the full possibility of the modern spectroscopic methods. For this reason, total synthesis has played a major role in the structure elucidation and revision of complex natural products for a long time. In the absence of a firm structural assignment, a combination of the stereochemical logic of the synthesis and spectroscopic comparison could be employed as tools to establish the correct structure of natural products [1-4]. Previous work in our group led to the reassignment of the configuration of a number of marine natural products [5-11]. These results encouraged us to embark upon the synthesis of other natural products with unknown configurations. We describe herein the determination of the complete relative and absolute stereochemistries of (+)-laingolide A and the total synthesis of this material.

Laingolide A (1), along with madangolide (2), was isolated in 1999 from the marine cyanobacterium Lyngbya bouillonii collected in Papua New Guinea [12]. The very same blue-green algae also produced laingolide (3) (Scheme 1), which was disclosed as the first member of the novel macrolide family [13]. Additionally, another chlorinated analogue, laingolide B (4), was isolated in 2010 by Luesch and co-workers from the same species of bacteria collected in Apra Harbor, Guam [14]. The planar structures of the laingolides were established using a combination of detailed 1D and 2D NMR analysis. However, these macrolides underwent degradation over time, which hampered progress towards complete assignments of their absolute stereochemistry. In 2010, Gerwick and co-workers reported the isolation of palmyrolide A (5), a structurally closely related 15-member macrolide from a cyanobacterial assemblage comprised of Leptolyngbya and Oscillatoria species collected at Palmyra Atoll, south of Hawaii [6]. One of the absolute configurations present in palmyrolide A was correctly assigned upon its initial isolation [15], and the absolute configurations of the remaining stereocentres were later established via total syntheses [16-24]. The structurally intriguing laingolides have attracted considerable attention from the synthetic community [25-27]. In 2018, Dai and co-workers reported the first total synthesis of laingolide B and unambiguously assigned the absolute configuration as depicted in structure 4 [27]. Laingolide A (1) was isolated from the bacteria collected in a different location and eleven years before the isolation of laingolide B (4). Laingolide A contains 
an extra undefined stereogenic centre located at C-(7), whose absolute structure remained elusive. Furthermore, laingolide A (1) bears a close resemblance to palmyrolide A (5); specifically, these two macrolides contain a tert-butyl carbinol and a methyl group beta to the tert-butyl substituted stereocenter.<smiles>[R]C(CCC(C)CC(OC(=O)CC=CN(C)C(=O)C(C)CC(C)C)C(C)(C)C)CC(C)C</smiles>

$\mathrm{R}=\mathrm{H}$, Laingolide $\mathrm{A}(1)$

$\mathrm{R}=\mathrm{Me}$, Laingolide (3)<smiles>CC(C)C(=O)N(C)/C=C/CC(=O)O[C@@H]1C/C(=C/Cl)CCCC[C@H](C)C1(C)C</smiles>

Laingolide B (4)<smiles>CCC(C)CCC(C)C(=O)N(C)/C=C\C=C/CCC(C)C(=O)OC(CC(C)C)C(C)(C)C</smiles>

Madangolide (2)<smiles>C[C@@H](CC/C=C/N(C)C(=O)CCC[C@H](C)C(C)(C)C)C(=O)OCC(C)(C)C</smiles>

Scheme 1. Structures of laingolide A (1), madangolide (2), laingolide (3), laingolide B (4) and palmyrolide A (5).

It would be interesting to find out whether the absolute configurations of laingolide A (1) were likely the same based on the possible similar biogenesis to palmyrolide A (5). Through the completion of the synthesis of four diastereomers, we shall be able to provide conclusive evidence for the absolute and relative stereochemistry of laingolide A (1) [28].

Structurally, laingolide A features a 15-member macrocyclic core, which is composed of a sterically encumbered ester derived from a tert-butyl carbinol, a trans- $N$-methyl enamide subunit and two chiral methyl appendages. At the outset of this synthetic venture, our primary objective was to rapidly access four diastereomers (Scheme 2) and thus conclusively establish the absolute configuration of laingolide A. With this in mind, we opted for a modular and flexible approach as we pondered its retrosynthetic analysis (Supplementary Materials) (Scheme 2) [29-36]. We envisioned that the four diastereomers of laingolide A could be constructed from fragments $6,7,8$ and ent-8 via three key transformations, as illustrated in Scheme 2. This highly convergent strategy relied on a ring-closing metathesis (RCM) at C-12 and C-13 to deliver the macrocycle and olefin migration to forge the enamide moiety at the final stage [19,22,27]. Both copper-catalysed Kumada-type coupling [37] of cyclic sulfate esters and Julia-Kocienski olefination [38-40] were then employed to construct the RCM precursor. 

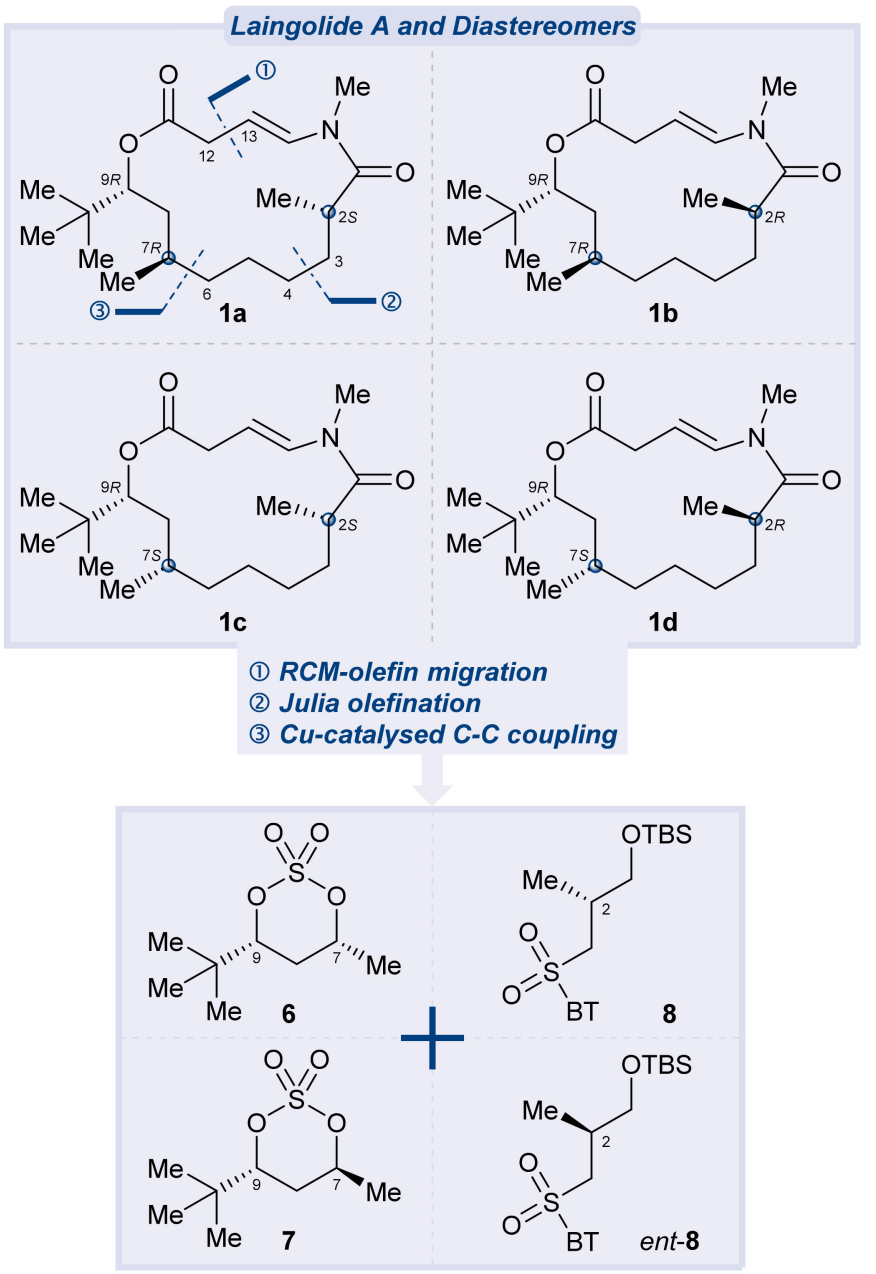

Scheme 2. Retrosynthetic analysis of laingolide A and its diastereomers (1a-d).

\section{Results}

The synthesis towards the chiral aldehydes (Scheme 3) commenced with the known chiral 1,3-hydroxy ketone 9 [23], which was prepared via List's proline-catalysed aldolisation between acetone and pivalaldehyde [41]. A three-step sequence [23] was employed to elaborate 1,3-hydroxy ketone 9 into cyclic sulfate ester 6 involving (1) the syn reduction of ketone 9 with DIBAL-H, (2) conversion of the syn-diol into the corresponding sulfite with thionyl chloride and (3) oxidation of the cyclic sulfite with $\mathrm{NaIO}_{4}$ in the presence of catalytic amounts of $\mathrm{RuCl}_{3}$. Nucleophilic ring-opening of the cyclic sulfate 6 using a mixed organometallic reagent derived from allylmagnesium chloride and stoichiometric quantities of copper(I) iodide has already been reported [42]. Recently, a catalytic version of this reaction, also termed $\mathrm{C}\left(\mathrm{sp}^{3}\right)-\mathrm{C}\left(\mathrm{sp}^{3}\right)$ Kumada-type coupling of cyclic sulfate esters was reported [37]. We opted to incorporate this catalytic reaction into our synthesis. Thus, treatment of cyclic sulfate 6 and $10 \mathrm{~mol} \%$ of cuprous iodide in THF with allyl magnesium bromide at $-20{ }^{\circ} \mathrm{C}$ followed by hydrolysis of the corresponding intermediate gave rise to the corresponding alcohol with a $75 \%$ yield with a $>95: 5$ diastereomeric ratio (dr), as determined using ${ }^{1} \mathrm{H}$ NMR spectroscopy. This reaction occurred at the least-hindered site, with the complete inversion of the configuration at that centre. Protection of the resulting alcohol with TBSOTf (tert-butyldimethylsilyl trifluoromethanesulfonate) and triethylamine afforded TBS ether $\mathbf{1 0}$ with a 96\% yield. Hydroboration of $\mathbf{1 0}$ with 9-borabicyclo[3.3.1] nonane $(9-\mathrm{BBN})$ and oxidation of the resulting organoborane $\left(\mathrm{NaHCO}_{3}, \mathrm{H}_{2} \mathrm{O}_{2}\right)$ furnished alcohol 11 with an 89\% yield, which in turn was subjected to oxidation with TEMPO, $\mathrm{NaOCl}$ and $\mathrm{NaBr}[43]$ to provide aldehyde 12 with an $85 \%$ yield. In parallel, the hydroxyl- 
directed antireduction of hydroxy ketone 9 with the Evans-Carreira protocol [44] proceeded smoothly to furnish the desired 1,3-anti diol with a diastereomeric ratio of 5:1 (determined by ${ }^{1} \mathrm{H}$ NMR of the crude reaction mixture). These diastereomers were separated using flash chromatography and the major one was used in subsequent reactions. The anti-substituted cyclic sulfate 7 was prepared with a $60 \%$ yield over three steps using the same procedure as described for $\mathbf{6}$. The elaboration of the substituted cyclic sulfate $\mathbf{7}$ into aldehyde $\mathbf{1 5}$ was accomplished in a way similar to that described for the preparation of aldehyde $\mathbf{1 2}$.

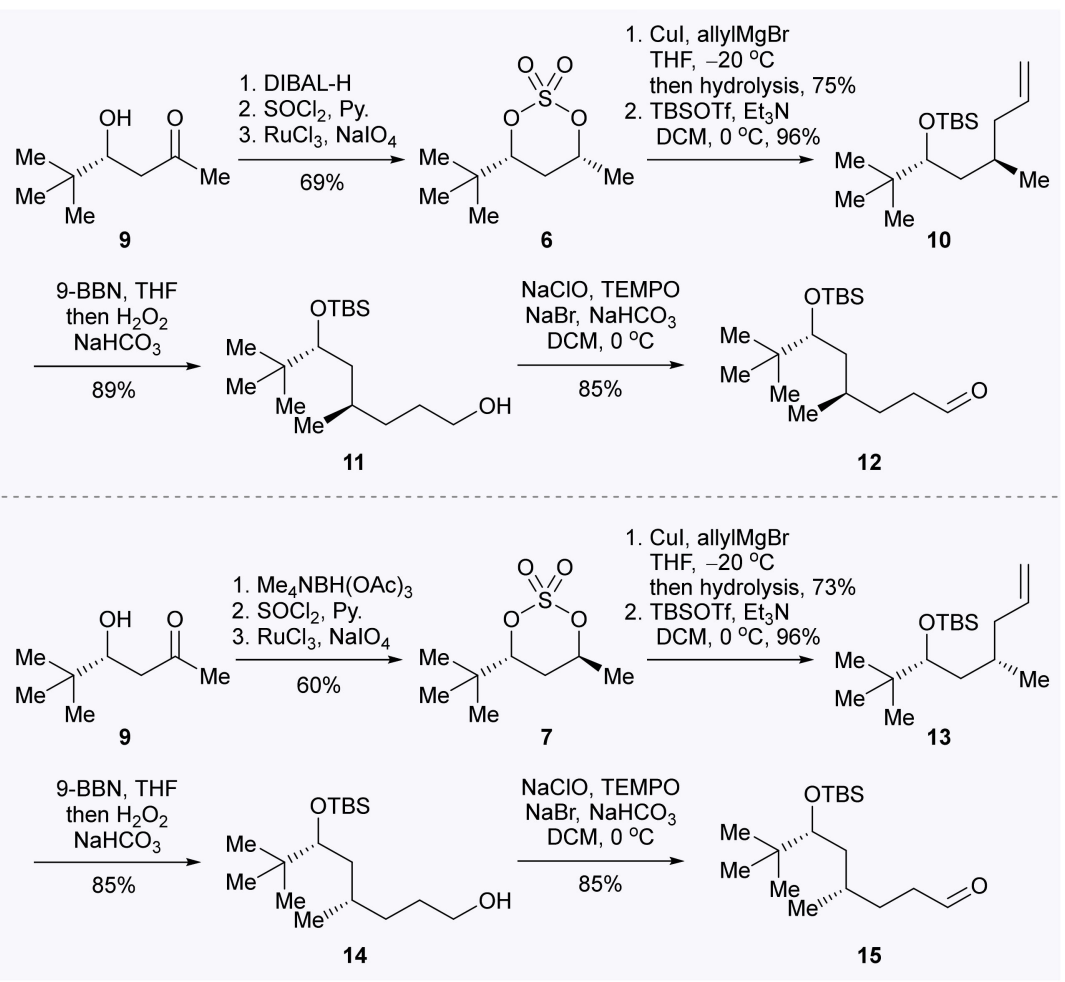

Scheme 3. Synthesis of aldehydes 12 and 15.

With a reliable route to useful quantities of the required aldehydes $\mathbf{1 2}$ and $\mathbf{1 5}$ in hand, our efforts turned to the divergent total synthesis of laingolide A (Scheme 4). This required the combination of aldehydes $\mathbf{1 2}$ and $\mathbf{1 5}$ separately with BT-sulfones $\mathbf{8}$ and then with ent-8 [45] (Scheme 4a). Under the optimum conditions investigated, each aldehyde (12 or 15) underwent condensation with 1.2 molar equivalent of sulfone (8 or ent-8) treated with 1.2 molar equivalent of NaHMDS in toluene at $-78^{\circ} \mathrm{C}$ to afford the corresponding alkene as a mixture of geometrical isomers $(Z: E \approx 3-7: 1)$ in high yield. Next, each of the resultant internal alkenes $(\mathbf{1 6}, \mathbf{2 0}, \mathbf{2 4}, \mathbf{2 8})$ was separately subjected to palladium-chloridemediated hydrogenation in ethanol with the concomitant removal of the TBS ethers that furnish the corresponding diol $(\mathbf{1 7}, \mathbf{2 1}, \mathbf{2 5}, \mathbf{2 9})$ [46]. The primary alcohol of the above diol was selectively oxidised with TEMPO in the presence of bis-acetoxyiodobenzene (BAIB) [47] and the resulting carboxylic acid was then coupled with the $N$-methylallylamine by utilising EDCI-HOAt and DMAP as a base to provide the corresponding amide (18, $22,26,30)$. For the conversion to the required diene $(19,23,27,31)$, each amide alcohol was separately acylated with acryloyl chloride in the presence of triethylamine and DMAP. The four diastereomeric dienes $(19,23,27,31)$ were separately subjected to ring-closing metathesis using the second-generation Grubbs catalyst (G-II) to afford the corresponding unsaturated macrolactone as isomeric mixtures, which were subsequently treated with $\mathrm{RuH}\left(\mathrm{PPh}_{3}\right)_{3}(\mathrm{CO}) \mathrm{Cl}$ in refluxing toluene [48] to furnish the desired enamides $\mathbf{1 a}-\mathbf{d}$ with good yields. The comparison of the spectral data of $\mathbf{1 a}-\mathbf{d}$ with the reported spectra of laingolide $\mathrm{A}$ was informative. Compound 1a, featuring a $\mathrm{C}(7)-R$ methyl beta to the $C(9)$ $R$-tert-butyl-substituted stereocenter, the same as that of natural palmyrolide A (5), did 
not match the literature values reported for the laingolide A. This suggested that the biogenesis of the laingolide A and palmyrolide A might follow different pathways or that C7 is epimerized at some stage of the biosynthesis of laingolide A (or of palmyrolide A). It was clear from comparing the ${ }^{13} \mathrm{C}$ NMR data (Scheme $4 \mathrm{~b}$ ) that diastereomer $\mathbf{1 c}$ represented the correct structure of natural laingolide A. The absolute stereochemical assignment of laingolide A was thus assigned as 2S,7S,9R, as shown in 1c (Scheme 4).

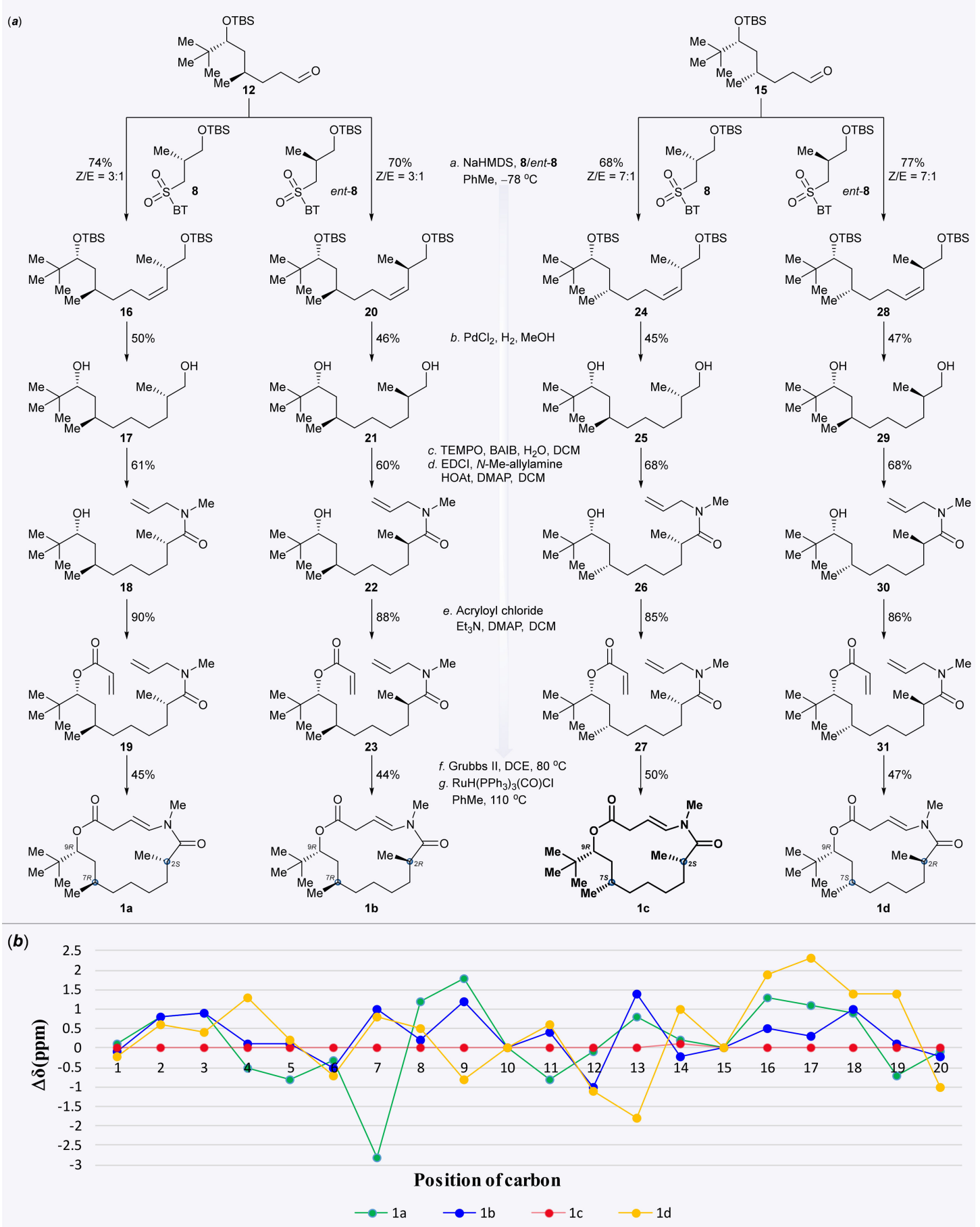

Scheme 4. (a) Total synthesis of laingolide A and its diastereomers (1a-d); (b) comparison of the ${ }^{13} \mathrm{C} N M R$ data of the synthetic compounds (1a-d) with the naturally occurring laingolide A. 


\section{Materials and Methods}

\subsection{General Information}

All reactions were conducted in flame-dried or oven-dried glassware under an atmosphere of dry nitrogen or argon. Oxygen and/or moisture-sensitive solids and liquids were transferred appropriately. The concentration of solutions in vacuo was accomplished using a rotary evaporator fitted with a water aspirator. Residual solvents were removed under a high vacuum $(0.1-0.2 \mathrm{~mm} \mathrm{Hg})$. All reaction solvents were purified before use: tetrahydrofuran (THF) was distilled from $\mathrm{Na}$ /benzophenone. Toluene was distilled over molten sodium metal. Dichloromethane (DCM), 1,2-dichloroethane (DCE) and trimethylamine $\left(\mathrm{Et}_{3} \mathrm{~N}\right)$ were distilled from $\mathrm{CaH}_{2}$. Methanol $(\mathrm{MeOH})$ was distilled from $\mathrm{Mg} / \mathrm{I}_{2}$. The reagents were purchased at the highest commercial quality and used without further purification, unless otherwise stated. Flash column chromatography was performed using the indicated solvents on E. Qingdao silica gel 60 (230-400 mesh ASTM). Reactions were monitored using thin-layer chromatography (TLC), which was carried out using pre-coated sheets (Qingdao silica gel 60-F250, 0.2 mm, Tsingtao, China). Compounds were visualised with UV light, iodine and ceric ammonium molybdate stainor phosphomolybdic acid in $\mathrm{EtOH}$. The ${ }^{1} \mathrm{H}$ NMR spectra were recorded on Bruker Avance $300 \mathrm{MHz}$, Avance $400 \mathrm{MHz}$ or Avance $500 \mathrm{MHz}$ spectrometers (Karlsruhe, Germany). Chemical shifts were reported in parts per million (ppm), relative to either a tetramethylsilane (TMS) internal standard or the signals due to the solvent (Supplementary Materials). The following abbreviations are used to describe the spin multiplicity: $\mathrm{s}=$ singlet, $\mathrm{d}=$ doublet, $\mathrm{t}=$ triplet, $\mathrm{q}=$ quartet, $\mathrm{qn}=$ quintet, $\mathrm{m}=$ multiplet, $\mathrm{br}=$ broad, $\mathrm{dd}=$ doublet of doublets, $\mathrm{dt}=$ doublet of triplets, $\mathrm{dq}=$ doublet of quartets, $\mathrm{ddd}=$ doublet of doublet of doublets; other combinations are derived from those listed above. Coupling constants $(J)$ are reported in hertz $(\mathrm{Hz})$ for corresponding solutions, and chemical shifts are reported as parts per million (ppm) relative to residual $\mathrm{CHCl}_{3} \delta \mathrm{H}(7.26 \mathrm{ppm}) .{ }^{13} \mathrm{C}$ Nuclear magnetic resonance spectra were recorded using a $75 \mathrm{MHz}, 100 \mathrm{MHz}$ or $125 \mathrm{MHz}$ spectrometer (Karlsruhe, Germany) for corresponding solutions, and chemical shifts are reported as parts per million (ppm) relative to residual $\mathrm{CDCl}_{3} \delta \mathrm{C}(77.16 \mathrm{ppm})$ (Supplementary Materials). High-resolution mass spectra were measured on an ABI Q-star Elite (Beijing, China). Optical rotations were recorded on a Rudolph AutoPol-I polarimeter (Shanghai, China) at $589 \mathrm{~nm}$ with a $50 \mathrm{~mm}$ cell. Data are reported as follows: specific rotation $(c(\mathrm{~g} / 100 \mathrm{~mL})$, solvent).

\subsection{General Experimental Procedures}

\subsubsection{Synthesis of (3R,5R)-2,2,5-trimethyloct-7-en-3-ol (S11)}

To a solution of cyclic sulfate $6(620 \mathrm{mg}, 2.98 \mathrm{mmol}, 1.0 \mathrm{eq}$.) and CuI $(57 \mathrm{mg}, 0.3 \mathrm{mmol}$, 0.1 eq. $)$ in dry THF $(1 \mathrm{~mL})$ at $-20{ }^{\circ} \mathrm{C}$, allylmagnesium bromide $(1.0 \mathrm{M}$ in THF, $14.9 \mathrm{~mL}$, $14.9 \mathrm{mmol}, 5.0$ eq.) was added under an argon atmosphere. The purple-colored reaction mixture was allowed to stir at $-20{ }^{\circ} \mathrm{C}$ for $7 \mathrm{~h}$ before it was allowed to warm to room temperature and then become concentrated in vacuo. The solid residue was redissolved in $\mathrm{Et}_{2} \mathrm{O}(30 \mathrm{~mL})$ and treated with $20 \%$ aqueous $\mathrm{H}_{2} \mathrm{SO}_{4}(10 \mathrm{~mL})$ solution. The contents of the flask were then stirred vigorously for another $12 \mathrm{~h}$ before the phases were separated. The aqueous layer was extracted with $\mathrm{Et}_{2} \mathrm{O}(3 \times 30 \mathrm{~mL})$. The combined organic layers were dried over anhydrous sodium sulfate, filtered and concentrated under reduced pressure. Purification of the crude product was performed using flash chromatography on silica gel (hexanes/EtOAc $=20: 1)$ to afford alcohol $\mathbf{S 1 1}(380 \mathrm{mg}, 75 \%)$ as a colorless oil. TLC: $\mathrm{R}_{f}=0.6$ (hexanes $/$ EtOAc $\left.=10: 1\right)$, iodine and PMA stain. $\alpha_{\mathrm{D}}^{23}=+36.0\left(\right.$ c 1.00, $\left.\mathrm{CHCl}_{3}\right) .{ }^{1} \mathrm{H}$ NMR $\left(500 \mathrm{MHz}, \mathrm{CDCl}_{3}\right) \delta 5.86-5.67(\mathrm{~m}, 1 \mathrm{H}), 5.14-4.91(\mathrm{~m}, 2 \mathrm{H}), 3.29(\mathrm{dd}, J=10.3,1.6 \mathrm{~Hz}$, $1 \mathrm{H}), 2.30-2.13(\mathrm{~m}, 1 \mathrm{H}), 1.92-1.82(\mathrm{~m}, 1 \mathrm{H}), 1.81-1.72(\mathrm{~m}, 1 \mathrm{H}), 1.53(\mathrm{~s}, 1 \mathrm{H}), 1.46-1.34(\mathrm{~m}$, $1 \mathrm{H}), 1.18(\mathrm{ddd}, J=14.3,10.3,4.1 \mathrm{~Hz}, 1 \mathrm{H}), 0.93(\mathrm{~d}, J=6.7 \mathrm{~Hz}, 3 \mathrm{H}), 0.87(\mathrm{~s}, 9 \mathrm{H}) ;{ }^{13} \mathrm{C} \mathrm{NMR}$ $\left(125 \mathrm{MHz}, \mathrm{CDCl}_{3}\right) \delta 137.2,116.1,77.7,39.9,38.6,35.1,29.9,25.7,21.0$; HRMS (ESI) calculated for $\mathrm{C}_{11} \mathrm{H}_{22} \mathrm{ONa}^{+}[\mathrm{M}+\mathrm{Na}]^{+}$193.1563, found 193.1565. 
3.2.2. Synthesis of tert-butyldimethyl(((3R,5R)-2,2,5-trimethyloct-7-en-3-yl)oxy)silane (10)

To a solution of alcohol $\mathbf{S 1 1}(2.8 \mathrm{~g}, 16.5 \mathrm{mmol}, 1.0$ eq. $)$ in dry DCM $(30 \mathrm{~mL}, 0.55 \mathrm{M})$, $\mathrm{Et}_{3} \mathrm{~N}(33 \mathrm{mmol}, 4.6 \mathrm{~mL}, 2.0$ eq.) and TBSOTf $(21.5 \mathrm{mmol}, 4.9 \mathrm{~mL}, 1.3$ eq.) were added at $0{ }^{\circ} \mathrm{C}$. The reaction mixture was allowed to stir at $0{ }^{\circ} \mathrm{C}$ for $2 \mathrm{~h}$ before it was diluted with DCM $(20 \mathrm{~mL})$ and quenched with a saturated aqueous solution of $\mathrm{NH}_{4} \mathrm{Cl}(30 \mathrm{~mL})$. The aqueous layer was extracted with DCM $(3 \times 50 \mathrm{~mL})$. The combined organic layers were washed with brine $(50 \mathrm{~mL})$, dried over anhydrous sodium sulfate, filtered and concentrated under reduced pressure. Purification of the crude product was performed using flash chromatography on silica gel (hexanes) to furnish silyl ether $10(4.5 \mathrm{~g}, 96 \%)$ as a colorless oil. TLC: $\mathrm{R}_{f}=0.95$ (hexanes), iodine and PMA stain. $\alpha_{\mathrm{D}}^{22}=+7.4\left(c 0.02, \mathrm{CHCl}_{3}\right) .{ }^{1} \mathrm{H} \mathrm{NMR}$ $\left(500 \mathrm{MHz}, \mathrm{CDCl}_{3}\right) \delta 5.83-5.73(\mathrm{~m}, 1 \mathrm{H}), 5.04-4.99(\mathrm{~m}, 2 \mathrm{H}), 3.34(\mathrm{dd}, J=7.5,2.8 \mathrm{~Hz}, 1 \mathrm{H})$, 2.22-2.16 (m, 1H), 1.80-1.72 (m, 1H), 1.72-1.64 (m, 1H), $1.45(\mathrm{ddd}, J=14.3,9.3,2.8 \mathrm{~Hz}, 1 \mathrm{H})$, $1.21(\mathrm{ddd}, J=14.3,7.5,4.2 \mathrm{~Hz}, 1 \mathrm{H}), 0.90(\mathrm{~d}, J=6.5 \mathrm{~Hz}, 3 \mathrm{H}), 0.90(\mathrm{~s}, 9 \mathrm{H}), 0.86(\mathrm{~s}, 9 \mathrm{H}), 0.07(\mathrm{~s}$, $3 \mathrm{H}), 0.06(\mathrm{~s}, 3 \mathrm{H}) ;{ }^{13} \mathrm{C}$ NMR $\left(125 \mathrm{MHz}, \mathrm{CDCl}_{3}\right) \delta 137.3,116.1,78.6,41.0,40.5,36.0,29.8,26.6$, 26.4, 20.9, 18.7, -3.2, -3.6; HRMS (ESI) calculated for $\mathrm{C}_{17} \mathrm{H}_{36} \mathrm{OSi} \mathrm{Na}^{+}[\mathrm{M}+\mathrm{Na}]^{+} 307.2428$, found 307.2425.

\subsubsection{Synthesis of (4R,6R)-6-((tert-butyldimethylsilyl)oxy)-4,7,7-trimethyloctan-1-ol (11)}

To a solution of alkene 10 ( $0.5 \mathrm{~g}, 1.7 \mathrm{mmol}, 1.0$ eq. $)$ in dry THF $(3 \mathrm{~mL}, 0.17 \mathrm{M}), 9-\mathrm{BBN}$ (0.5 M in THF, $3.52 \mathrm{mmol}, 7.0 \mathrm{~mL}, 2.0$ eq.) was added at $0{ }^{\circ} \mathrm{C}$ under an argon atmosphere. The reaction mixture was stirred at room temperature for $8 \mathrm{~h}$ before a saturated aqueous solution of $\mathrm{NaHCO}_{3}(10 \mathrm{~mL})$ and $30 \% \mathrm{H}_{2} \mathrm{O}_{2}(2 \mathrm{~mL})$ were added sequentially at $0{ }^{\circ} \mathrm{C}$ and stirred for another $12 \mathrm{~h}$ at room temperature. The aqueous layer was extracted with EtOAc $(3 \times 10 \mathrm{~mL})$. The combined organic layers were washed with brine $(10 \mathrm{~mL})$, dried over anhydrous sodium sulfate, filtered and concentrated under reduced pressure. Purification of the crude product was performed using flash chromatography on silica gel (hexanes $/ \mathrm{EtOAc}=10: 1)$ to afford alcohol $11(473 \mathrm{mg}, 89 \%)$ as a colorless oil. TLC: $\mathrm{R}_{f}=0.5$ (hexanes $/ \mathrm{EtOAc}=4: 1)$, PMA stain. $\alpha_{\mathrm{D}}^{24}=+10.8\left(c 0.01, \mathrm{CHCl}_{3}\right) ;{ }^{1} \mathrm{H} \mathrm{NMR}\left(400 \mathrm{MHz}, \mathrm{CDCl}_{3}\right)$ $\delta 3.63(\mathrm{td}, \mathrm{J}=6.6,1.5 \mathrm{~Hz}, 2 \mathrm{H}), 3.30(\mathrm{dd}, J=7.3,2.9 \mathrm{~Hz}, 1 \mathrm{H}), 1.73-1.58(\mathrm{~m}, 1 \mathrm{H}), 1.59-1.53$ $(\mathrm{m}, 1 \mathrm{H}), 1.52-1.36(\mathrm{~m}, 4 \mathrm{H}), 1.19(\mathrm{ddd}, J=14.2,7.3,4.4 \mathrm{~Hz}, 1 \mathrm{H}), 1.06-0.94(\mathrm{~m}, 1 \mathrm{H}), 0.90(\mathrm{~d}$, $J=6.6 \mathrm{~Hz}, 3 \mathrm{H}), 0.88(\mathrm{~s}, 9 \mathrm{H}), 0.84(\mathrm{~s}, 9 \mathrm{H}), 0.04(\mathrm{~s}, 6 \mathrm{H}) ;{ }^{13} \mathrm{C} \mathrm{NMR}\left(100 \mathrm{MHz}, \mathrm{CDCl}_{3}\right) \delta 78.5$, $63.7,41.7,35.9,32.2,30.3,29.9,26.5,26.3,20.9,18.6,-3.3,-3.7$; HRMS (ESI) calculated for $\mathrm{C}_{17} \mathrm{H}_{38} \mathrm{O}_{2} \mathrm{SiNa}^{+}[\mathrm{M}+\mathrm{Na}]^{+} 325.2533$, found 325.2528.

\subsubsection{Synthesis of (4R,6R)-6-((tert-butyldimethylsilyl)oxy)-4,7,7-trimethyloctanal (12)}

To a solution of alcohol 11 (1.0 g, $3.3 \mathrm{mmol}, 1.0$ eq.) and TEMPO $(51 \mathrm{mg}, 0.33 \mathrm{mmol}$, 0.1 eq.) in $\mathrm{DCM}(30 \mathrm{~mL})$, a solution of $\mathrm{NaBr}\left(2.0 \mathrm{~g}, 19.8 \mathrm{mmol}, 6.0\right.$ eq.) and $\mathrm{NaHCO}_{3}(1.7 \mathrm{~g}$, $19.8 \mathrm{mmol}$, 6.0 eq.) were added in water $(50 \mathrm{~mL})$, followed by $\mathrm{NaClO}(1 \mathrm{M}, 3.3 \mathrm{~mL}, 1.0$ eq.) at $0{ }^{\circ} \mathrm{C}$. The reaction mixture was stirred at $0{ }^{\circ} \mathrm{C}$ for $10 \mathrm{~min}$ and then quenched with a saturated aqueous solution of $\mathrm{Na}_{2} \mathrm{~S}_{2} \mathrm{O}_{3}(3 \mathrm{~mL})$ and extracted with $\mathrm{DCM}(3 \times 30 \mathrm{~mL})$. The combined organic layers were washed with brine $(30 \mathrm{~mL})$, dried over anhydrous sodium sulfate, filtered and concentrated under reduced pressure. Purification of the crude product was performed using flash chromatography on silica gel (hexanes $/ \mathrm{EtOAc}=20: 1$ ) to afford aldehyde $12(842 \mathrm{mg}, 85 \%)$ as a colorless oil. TLC: $\mathrm{R}_{f}=0.6$ (hexanes $/$ EtOAc $\left.=10: 1\right)$, PMA stain. $\alpha_{\mathrm{D}}^{27}=+11.5\left(\mathrm{c} 0.01, \mathrm{CHCl}_{3}\right) .{ }^{1} \mathrm{H} \mathrm{NMR}\left(500 \mathrm{MHz}, \mathrm{CDCl}_{3}\right) \delta 9.78(\mathrm{t}, J=1.8 \mathrm{~Hz}, 1 \mathrm{H}), 3.30$ $(\mathrm{dd}, J=7.3,2.9 \mathrm{~Hz}, 1 \mathrm{H}), 2.58-2.44(\mathrm{~m}, 1 \mathrm{H}), 2.40-2.29(\mathrm{~m}, 1 \mathrm{H}), 1.90-1.71(\mathrm{~m}, 1 \mathrm{H}), 1.65-1.53$ $(\mathrm{m}, 1 \mathrm{H}), 1.44(\mathrm{ddd}, J=14.3,9.0,2.8 \mathrm{~Hz}, 1 \mathrm{H}), 1.34-1.25(\mathrm{~m}, 1 \mathrm{H}), 1.22(\mathrm{ddd}, J=14.3,7.1$, $4.3 \mathrm{~Hz}, 1 \mathrm{H}), 0.90(\mathrm{~d}, J=6.7 \mathrm{~Hz}, 3 \mathrm{H}), 0.88(\mathrm{~s}, 9 \mathrm{H}), 0.84(\mathrm{~s}, 9 \mathrm{H}), 0.04(\mathrm{~s}, 3 \mathrm{H}), 0.02(\mathrm{~s}, 3 \mathrm{H}) ;{ }^{13} \mathrm{C}$ NMR (125 MHz, $\left.\mathrm{CDCl}_{3}\right) \delta$ 202.7, 78.4, 41.6, 41.5, 36.0, 29.7, 28.0, 26.5, 26.3, 20.7, 18.6, -3.3, -3.7; HRMS (ESI) calculated for $\mathrm{C}_{17} \mathrm{H}_{38} \mathrm{O}_{2} \mathrm{SiNa}^{+}[\mathrm{M}+\mathrm{Na}]^{+} 323.2377$, found 323.2367.

3.2.5. Synthesis of (4R,6S)-4-(tert-butyl)-6-methyl-1,3,2-dioxathiane 2,2-dioxide (7)

To a solution of $\mathrm{Me}_{4} \mathrm{NHB}(\mathrm{OAc})_{3}\left(13.7 \mathrm{~g}\right.$, $52.1 \mathrm{mmol}, 5.0$ eq.) in anhydrous $\mathrm{CH}_{3} \mathrm{CN}$ $(25 \mathrm{~mL})$ and anhydrous $\mathrm{AcOH}(15 \mathrm{~mL})$ at $-40^{\circ} \mathrm{C}$, a solution of 9 [23] $(1.5 \mathrm{~g}, 10.4 \mathrm{mmol}$, 
1.0 eq.) was added in anhydrous $\mathrm{CH}_{3} \mathrm{CN}(15 \mathrm{~mL})$. The reaction mixture was stirred at $-40{ }^{\circ} \mathrm{C}$ for $12 \mathrm{~h}$, allowed to warm to ambient temperature, poured into a saturated aqueous solution of $\mathrm{Na}_{2} \mathrm{CO}_{3}(80 \mathrm{~mL})$ and then extracted with EtOAc $(3 \times 100 \mathrm{~mL})$. The combined organic layers were washed with brine $(10 \mathrm{~mL})$, dried over anhydrous sodium sulfate, filtered and concentrated under reduced pressure. Purification of the crude product was performed using flash chromatography on silica gel (hexanes/EtOAc $=10: 1-5: 1$ ) to afford diol S13 $(1.1 \mathrm{~g}, 71 \%)$ as a white solid. [43] ${ }^{1} \mathrm{H}$ NMR analysis revealed the presence of a 5:1 ratio of anti/syn.

To a solution of anti-diol S13 (3.15 g, $21.5 \mathrm{mmol}, 1.0$ eq.) in dry DCM (200 mL, $0.22 \mathrm{M})$, pyridine (17.3 mL, $215.0 \mathrm{mmol}, 10.0$ eq.) and $\mathrm{SOCl}_{2}(7.9 \mathrm{~mL}, 108.0 \mathrm{mmol}, 5.0$ eq.) were added sequentially at $0{ }^{\circ} \mathrm{C}$. The reaction mixture was allowed to stir at $0{ }^{\circ} \mathrm{C}$ for $45 \mathrm{~min}$ before it was quenched by the addition of water $(50 \mathrm{~mL})$ and then extracted with DCM $(3 \times 100 \mathrm{~mL})$. The combined organic layers were washed with saturated aqueous $\mathrm{KHSO}_{4}$ solution $(50 \mathrm{~mL})$, followed by saturated aqueous $\mathrm{NaHCO}_{3}$ solution $(60 \mathrm{~mL})$, dried over anhydrous sodium sulfate, filtered and concentrated under reduced pressure to afford the crude sulfite, which was used in the next step without further purification. To a solution of the crude sulfite in a mixture of $\mathrm{H}_{2} \mathrm{O} / \mathrm{MeCN} / \mathrm{CCl}_{4}(200 \mathrm{~mL}: 200 \mathrm{~mL}: 100 \mathrm{~mL}), \mathrm{RuCl}_{3} \cdot \mathrm{nH}_{2} \mathrm{O}$ (562 mg, $2.15 \mathrm{mmol}, 0.1$ eq.) and $\mathrm{NaIO}_{4}(6.9 \mathrm{~g}, 32.3 \mathrm{mmol}, 1.5$ eq.) were added. The biphasic reaction mixture was vigorously stirred at room temperature for $2 \mathrm{~h}$ before it was diluted with $\mathrm{Et}_{2} \mathrm{O}(60 \mathrm{~mL})$ and quenched with a saturated aqueous solution of $\mathrm{NaHCO}_{3}$ $(100 \mathrm{~mL})$. The aqueous layer was extracted with $\mathrm{Et}_{2} \mathrm{O}(3 \times 200 \mathrm{~mL})$. The combined organic layers were dried over anhydrous sodium sulfate, filtered and concentrated under reduced pressure. Purification of the crude product was performed using flash chromatography on silica gel (hexanes/EtOAc $=10: 1-5: 1)$ to afford anti-cyclic sulfate 7 (3.76 g, 84\% for two steps) as an amorphous white solid. The spectral data are in accordance with those reported in literature for its enantiomer [23]. TLC: $\mathrm{R}_{f}=0.5$ (hexanes $/$ EtOAC $=4: 1$ ), PMA stain. $\alpha_{\mathrm{D}}^{25}=-0.06\left(\mathrm{c} 0.03, \mathrm{CHCl}_{3}\right) .{ }^{1} \mathrm{H} \mathrm{NMR}\left(400 \mathrm{MHz}, \mathrm{CDCl}_{3}\right) \delta 4.94(\mathrm{ddq}, J=6.7,6.1$, $4.4 \mathrm{~Hz}, 1 \mathrm{H}), 4.59(\mathrm{dd}, J=11.4,3.6 \mathrm{~Hz}, 1 \mathrm{H}), 2.30(\mathrm{ddd}, J=14.2,11.4,6.1 \mathrm{~Hz}, 1 \mathrm{H}), 1.75(\mathrm{ddd}$, $J=14.2,4.4,3.6 \mathrm{~Hz}, 1 \mathrm{H}), 1.63(\mathrm{~d}, J=6.8 \mathrm{~Hz}, 3 \mathrm{H}), 1.00(\mathrm{~s}, 9 \mathrm{H}) .{ }^{13} \mathrm{C}$ NMR $\left(100 \mathrm{MHz}, \mathrm{CDCl}_{3}\right)$ $\delta$ 88.6, 81.1, 34.3, 30.0, 25.2, 19.7. HRMS (ESI) calculated for $\mathrm{C}_{8} \mathrm{H}_{16} \mathrm{SO}_{4} \mathrm{Na}^{+}[\mathrm{M}+\mathrm{Na}]^{+}$ 231.0662 , found 231.0661 .

\subsubsection{Synthesis of (3R,5S)-2,2,5-trimethyloct-7-en-3-ol (S12)}

The product $\mathbf{S 1 2}$ was synthesised according to the procedures for the synthesis of S11 from 7 ( $620 \mathrm{mg}, 2.98 \mathrm{mmol}, 1.0$ eq.). Purification of the crude product was performed using flash chromatography on silica gel (hexanes $/ \mathrm{EtOAc}=20: 1)$ to afford $\mathbf{S 1 2}(370 \mathrm{mg}, 73 \%)$ as a colorless oil. TLC: $\mathrm{R}_{f}=0.6$ (hexanes $/$ EtOAc $=10: 1$ ), iodine and PMA stain. $\alpha_{\mathrm{D}}^{25}=+24.9$ (c $\left.0.01, \mathrm{CHCl}_{3}\right) .{ }^{1} \mathrm{H}$ NMR $\left(400 \mathrm{MHz}, \mathrm{CDCl}_{3}\right) \delta 5.78(\mathrm{ddt}, J=17.4,10.4,7.1 \mathrm{~Hz}, 1 \mathrm{H}), 5.13-4.81$ $(\mathrm{m}, 2 \mathrm{H}), 3.28(\mathrm{dd}, J=10.6,1.8 \mathrm{~Hz}, 1 \mathrm{H}), 2.21-1.85(\mathrm{~m}, 2 \mathrm{H}), 1.75(\mathrm{dtt}, J=13.6,6.7,3.3 \mathrm{~Hz}, 1 \mathrm{H})$, 1.35 (ddd, $J=13.9,10.6,3.2 \mathrm{~Hz}, 1 \mathrm{H}), 1.18(\mathrm{ddd}, J=14.0,10.6,1.8 \mathrm{~Hz}, 1 \mathrm{H}), 0.89(\mathrm{~d}, J=6.5 \mathrm{~Hz}$, 3H), 0.87 (s, 9H). ${ }^{13} \mathrm{C}$ NMR $\left(100 \mathrm{MHz}, \mathrm{CDCl}_{3}\right) \delta$ 137.6, 115.9, 77.4, 42.8, 38.5, 35.0, 29.7, 25.8, 18.9. HRMS (ESI) calculated for $\mathrm{C}_{11} \mathrm{H}_{22} \mathrm{ONa}^{+}[\mathrm{M}+\mathrm{Na}]^{+} 193.1563$, found 193.1562 .

3.2.7. Synthesis of tert-butyldimethyl(((3R,5S)-2,2,5-trimethyloct-7-en-3-yl)oxy)silane (13)

The product $\mathbf{1 3}$ was synthesised according to the procedures for the synthesis of $\mathbf{1 0}$ from S12 $(2.9 \mathrm{~g}, 17.14 \mathrm{mmol}, 1.0 \mathrm{eq}$.$) . Purification of the crude product was performed$ using flash chromatography on silica gel (hexanes) to afford $13(4.67 \mathrm{~g}, 96 \%)$ as a colorless oil. TLC: $\mathrm{R}_{f}=0.95$ (hexanes), iodine and PMA stain. $\alpha_{\mathrm{D}}^{27}=+11.1\left(c 0.01, \mathrm{CHCl}_{3}\right) .{ }^{1} \mathrm{H} \mathrm{NMR}$ $\left(400 \mathrm{MHz}, \mathrm{CDCl}_{3}\right) \delta 5.98-5.61(\mathrm{~m}, 1 \mathrm{H}), 5.19-4.90(\mathrm{~m}, 2 \mathrm{H}), 3.30(\mathrm{dd}, J=8.5,1.8 \mathrm{~Hz}, 1 \mathrm{H})$, 2.12-1.97 (m, 1H), 1.96-1.84 (m, 1H), 1.75-1.62 (m, 1H), $1.38(\mathrm{ddd}, J=14.1,8.5,2.9 \mathrm{~Hz}, 1 \mathrm{H})$, $1.19(\mathrm{ddd}, J=14.0,10.9,1.8 \mathrm{~Hz}, 1 \mathrm{H}), 0.89(\mathrm{~s}, 8 \mathrm{H}), 0.84(\mathrm{~d}, J=6.5 \mathrm{~Hz}, 3 \mathrm{H}), 0.84(\mathrm{~s}, 9 \mathrm{H}), 0.04(\mathrm{~s}$, $6 \mathrm{H}) .{ }^{13} \mathrm{C}$ NMR $\left(100 \mathrm{MHz}, \mathrm{CDCl}_{3}\right) \delta$ 137.7, 115.7, 78.6, 43.1, 40.6, 35.8, 29.6, 26.6, 26.4, 19.2, 18.7, $-3.2,-3.5$. HRMS (ESI) calculated for $\mathrm{C}_{17} \mathrm{H}_{36} \mathrm{OSi} \mathrm{Na}{ }^{+}[\mathrm{M}+\mathrm{Na}]^{+} 307.2428$, found 307.2423 . 


\subsubsection{Synthesis of (4S,6R)-6-((tert-butyldimethylsilyl)oxy)-4,7,7-trimethyloctan-1-ol (14)}

The product $\mathbf{1 4}$ was synthesised according to the procedures for the synthesis of $\mathbf{1 1}$ from $13(2.58 \mathrm{~g}, 9.08 \mathrm{mmol}, 1.0$ eq.). Purification of the crude product was performed using flash chromatography on silica gel (hexanes $/ \mathrm{EtOAc}=10: 1)$ to afford alcohol $14(2.33 \mathrm{~g}$, $85 \%)$ as a colorless oil. TLC: $\mathrm{R}_{f}=0.5$ (hexanes $/$ EtOAc $\left.=4: 1\right)$, PMA stain. $\alpha_{\mathrm{D}}^{28}=+11.1(c 0.01$, $\left.\mathrm{CHCl}_{3}\right) .{ }^{1} \mathrm{H}$ NMR $\left(400 \mathrm{MHz}, \mathrm{CDCl}_{3}\right) \delta 3.62(\mathrm{td}, J=6.8,1.1 \mathrm{~Hz}, 2 \mathrm{H}), 3.29(\mathrm{dd}, J=8.6,1.7 \mathrm{~Hz}$, $1 \mathrm{H}), 1.65-1.48(\mathrm{~m}, 4 \mathrm{H}), 1.36(\mathrm{ddd}, J=14.0,8.6,2.8 \mathrm{~Hz}, 1 \mathrm{H}), 1.25(\mathrm{ddd}, J=7.3,6.2,2.5 \mathrm{~Hz}$, $1 \mathrm{H}), 1.25-1.12(\mathrm{~m}, 2 \mathrm{H}), 0.89(\mathrm{~s}, 9 \mathrm{H}), 0.84(\mathrm{~d}, J=7.3 \mathrm{~Hz}, 3 \mathrm{H}), 0.83(\mathrm{~s}, 9 \mathrm{H}), 0.04(\mathrm{~s}, 3 \mathrm{H}), 0.03$ (s, 3H). ${ }^{13} \mathrm{C}$ NMR $\left(100 \mathrm{MHz}, \mathrm{CDCl}_{3}\right) \delta 78.6,63.5,41.0,35.7,34.7,30.5,29.3,26.6,26.4,19.4$, 18.7, $-3.1,-3.5$. HRMS (ESI) calculated for $\mathrm{C}_{17} \mathrm{H}_{38} \mathrm{O}_{2} \mathrm{SiNa}^{+}[\mathrm{M}+\mathrm{Na}]^{+} 325.2533$, found 325.2534 .

\subsubsection{Synthesis of (4S,6R)-6-((tert-butyldimethylsilyl)oxy)-4,7,7-trimethyloctanal (15)}

The product $\mathbf{1 5}$ was synthesised according to the procedures for the synthesis of $\mathbf{1 2}$ from $14(2.0 \mathrm{~g}, 6.6 \mathrm{mmol}, 1.0 \mathrm{eq}$.$) . Purification of the crude product was performed using$ flash chromatography on silica gel (hexanes $/$ EtOAc $=20: 1)$ to afford aldehyde $15(1.68 \mathrm{~g}$, $85 \%$ ) as a colorless oil. TLC: $R_{f}=0.6$ (hexanes $/$ EtOAc $=10: 1$ ), PMA stain. $\alpha_{\mathrm{D}}^{25}=+12.7$ (c 0.01, $\left.\mathrm{CHCl}_{3}\right) .{ }^{1} \mathrm{H}$ NMR $\left(400 \mathrm{MHz}, \mathrm{CDCl}_{3}\right) \delta 9.76(\mathrm{t}, J=1.8 \mathrm{~Hz}, 1 \mathrm{H}), 3.29(\mathrm{dd}, J=8.6$, $1.7 \mathrm{~Hz}, 1 \mathrm{H}), 2.51-2.30(\mathrm{~m}, 2 \mathrm{H}), 1.68-1.43(\mathrm{~m}, 3 \mathrm{H}), 1.35(\mathrm{ddd}, J=13.9,8.6,2.5 \mathrm{~Hz}, 1 \mathrm{H}), 1.20$ $(\mathrm{ddd}, J=14.0,10.4,1.8 \mathrm{~Hz}, 1 \mathrm{H}), 0.88(\mathrm{~s}, 9 \mathrm{H}), 0.85(\mathrm{~d}, J=6.1 \mathrm{~Hz}, 3 \mathrm{H}), 0.83(\mathrm{~s}, 9 \mathrm{H}), 0.03(\mathrm{~s}$, $3 \mathrm{H}), 0.02(\mathrm{~s}, 3 \mathrm{H}) .{ }^{13} \mathrm{C} \mathrm{NMR}\left(100 \mathrm{MHz}, \mathrm{CDCl}_{3}\right) \delta 202.9,78.4,41.9,40.7,35.7,30.5,29.2,26.5$, 26.4, 19.2, 18.7, -3.2, -3.5. HRMS (ESI) calculated for $\mathrm{C}_{17} \mathrm{H}_{38} \mathrm{O}_{2} \mathrm{SiNa}^{+}[\mathrm{M}+\mathrm{Na}]^{+} 323.2377$, found 323.2376 .

3.2.10. Synthesis of (6S,11R,13R,Z)-13-(tert-butyl)-2,2,3,3,6,11,15,15,16,16-decamethyl-4,14dioxa-3,15-disilaheptadec-7-ene (16)

To a cooled $\left(-78^{\circ} \mathrm{C}\right)$ stirring solution of sulfone 8 [44] $(200 \mathrm{mg}, 0.52 \mathrm{mmol}, 1.2 \mathrm{eq}$.$) in$ dry toluene ( $4 \mathrm{~mL}, 0.1 \mathrm{M})$, NaHMDS ( $2 \mathrm{M}$ in THF, $0.26 \mathrm{~mL}, 0.52 \mathrm{mmol}, 1.2 \mathrm{eq}$.) was added dropwise for $1 \mathrm{~h}$, followed by a solution of aldehyde $12(130 \mathrm{mg}, 0.43 \mathrm{mmol}, 1.0 \mathrm{eq}$.) in dry toluene $(2 \mathrm{~mL}, 0.22 \mathrm{M})$. The reaction mixture was stirred at $-78{ }^{\circ} \mathrm{C}$ for $3 \mathrm{~h}$ and then quenched with a saturated aqueous solution of $\mathrm{NH}_{4} \mathrm{Cl}(10 \mathrm{~mL})$. The layers were separated and the aqueous layer was extracted with MTBE $(3 \times 15 \mathrm{~mL})$. The combined organic layers were dried over anhydrous sodium sulfate, filtered and concentrated under reduced pressure. Purification of the crude product was performed using flash chromatography on silica gel (hexanes $/ \mathrm{EtOAc}=100: 1)$ to afford olefin $16(149 \mathrm{mg}, 74 \%)$ as a colorless oil. TLC: $\mathrm{R}_{f}=0.6$ (hexanes $/$ EtOAc $=80: 1$ ), iodine and PMA stain. $\alpha_{\mathrm{D}}^{24}=+15.8\left(c 0.01, \mathrm{CHCl}_{3}\right) .{ }^{1} \mathrm{H}$ NMR $\left(400 \mathrm{MHz}, \mathrm{CDCl}_{3}\right.$ as a mixture of $\left.Z / E=3: 1\right) \delta(5.53-5.27(\mathrm{~m})$ and $5.14(\mathrm{ddt}, J=11.0$, 9.5, 1.6 Hz), 2H), $3.47(\mathrm{ddd}, J=9.7,6.0,4.9 \mathrm{~Hz}, 1 \mathrm{H}), 3.35(\mathrm{ddd}, J=9.8,7.3,5.3 \mathrm{~Hz}, 1 \mathrm{H}), 3.30$ $(\mathrm{dd}, J=7.1,2.9 \mathrm{~Hz}, 1 \mathrm{H}),(2.69-2.54(\mathrm{~m})$ and $2.33-2.21(\mathrm{~m}), 1 \mathrm{H}), 2.21-2.08(\mathrm{~m}, 1 \mathrm{H}), 2.12-1.98$ $(\mathrm{m}, 1 \mathrm{H}), 2.02-1.86(\mathrm{~m}, 1 \mathrm{H}), 1.64-1.51(\mathrm{~m}, 1 \mathrm{H}), 1.52-1.37(\mathrm{~m}, 2 \mathrm{H}), 1.24-1.12(\mathrm{~m}, 1 \mathrm{H}), 1.09-0.97$ $(\mathrm{m}, 1 \mathrm{H}),(0.97(\mathrm{~d}, J=6.8 \mathrm{~Hz})$ and $0.96(\mathrm{~d}, J=6.7 \mathrm{~Hz}), 3 \mathrm{H}), 0.92(\mathrm{~d}, J=6.6 \mathrm{~Hz}, 3 \mathrm{H}), 0.90(\mathrm{~m}$, $18 \mathrm{H}), 0.85(\mathrm{~s}, 9 \mathrm{H}), 0.09(\mathrm{~m}, 12 \mathrm{H}) .{ }^{13} \mathrm{C}$ NMR $\left(100 \mathrm{MHz}, \mathrm{CDCl}_{3}\right) \delta 132.8,132.6,130.5,130.4$, 78.7, 78.6, 68.5, 68.2, 41.9, 41.8, 39.5, 36.7, 36.3, 36.0, 35.0, 30.2, 30.0, 29.7, 26.5, 26.4, 26.2, 25.1, 20.9, 20.8, 18.7, 18.6, 17.7, 16.9, -3.2, -3.7, -5.1, -5.1. HRMS (ESI) calculated for $\mathrm{C}_{27} \mathrm{H}_{58} \mathrm{O}_{2} \mathrm{Si}_{2} \mathrm{Na}^{+}[\mathrm{M}+\mathrm{Na}]^{+} 493.3869$, found 493.3869 .

\subsubsection{Synthesis of (2S,7R,9R)-2,7,10,10-tetramethylundecane-1,9-diol (17)}

To a solution of alkene 16 (149 mg, $0.317 \mathrm{mmol}, 1.0$ eq.) in anaerobic $\mathrm{MeOH}(10 \mathrm{~mL}$, $0.03 \mathrm{M}), \mathrm{PdCl}_{2}(17 \mathrm{mg}, 0.095 \mathrm{mmol}, 0.3$ eq.) was added under an argon atmosphere. The reaction flask was evacuated and purged with $\mathrm{H}_{2}$ three times and then the reaction was stirred at ambient temperature under a hydrogen atmosphere for $10 \mathrm{~h}$. The reaction flask was then evacuated and purged with nitrogen three times. The catalyst was removed via filtration through Celite. The filter cake was rinsed thoroughly with $\mathrm{MeOH}$ and the filtrate 
was concentrated in vacuo to provide the crude product. Purification of the crude product was performed using flash chromatography on silica gel (hexanes/EtOAc $=3: 1$ ) to afford diol 17 (39 mg, 50\%) as a colorless oil. TLC: $\mathrm{R}_{f}=0.3$ (hexanes/ EtOAc $=3: 1$ ), PMA stain. $\alpha_{\mathrm{D}}^{26}=+22.6\left(c 0.01, \mathrm{CHCl}_{3}\right) .{ }^{1} \mathrm{H} \mathrm{NMR}\left(500 \mathrm{MHz}, \mathrm{CDCl}_{3}\right) \delta 3.48(\mathrm{dd}, J=10.5,5.8 \mathrm{~Hz}, 1 \mathrm{H})$, $3.42(\mathrm{dd}, J=10.5,6.3 \mathrm{~Hz}, 1 \mathrm{H}), 3.28(\mathrm{dd}, J=10.2,1.8 \mathrm{~Hz}, 1 \mathrm{H}), 1.65-1.57(\mathrm{~m}, 3 \mathrm{H}), 1.45-1.39(\mathrm{~m}$, 2H), 1.38-1.33 (m, 3H), 1.25-1.19 (m, 3H), 1.18-1.14 (m, 1H), 1.11-1.05 (m, 1H), 1.03-0.99 $(\mathrm{m}, 1 \mathrm{H}), 0.92(\mathrm{~d}, J=6.7 \mathrm{~Hz}, 3 \mathrm{H}), 0.90(\mathrm{~d}, J=6.8 \mathrm{~Hz}, 3 \mathrm{H}) 0.87(\mathrm{~s}, 9 \mathrm{H}) .{ }^{13} \mathrm{C} \mathrm{NMR}(125 \mathrm{MHz}$, $\left.\mathrm{CDCl}_{3}\right) \delta 77.7,68.4,39.4,35.9,35.4,35.1,33.2,29.9,27.4,27.1,25.8,21.2,16.8$. HRMS (ESI) calculated for $\mathrm{C}_{15} \mathrm{H}_{32} \mathrm{O}_{2} \mathrm{Na}^{+}[\mathrm{M}+\mathrm{Na}]^{+}$267.2295, found 267.2293.

3.2.12. Synthesis of (2S,7R,9R)-9-hydroxy-2,7,10,10-tetramethylundecanoic acid (S14)

To a solution of diol 17 (53 mg, $0.217 \mathrm{mmol}, 1.0$ eq.) in DCM (3 mL, 0.07 M), TEMPO (7 mg, $0.043 \mathrm{mmol}, 0.2$ eq.), $\mathrm{H}_{2} \mathrm{O}$ (0.2 mL, $11.0 \mathrm{mmol}, 50$ eq.) and $\mathrm{PhI}(\mathrm{OAc})_{2}(175 \mathrm{mg}$, $0.54 \mathrm{mmol}, 2.5$ eq.) were sequentially added and stirred at room temperature for $20 \mathrm{~h}$ before it was quenched with saturated aqueous solution of $\mathrm{Na}_{2} \mathrm{~S}_{2} \mathrm{O}_{3}(3 \mathrm{~mL})$ and extracted with DCM $(3 \times 10 \mathrm{~mL})$. The combined organic layers were washed with brine $(10 \mathrm{~mL})$, dried over anhydrous sodium sulfate, filtered and concentrated under reduced pressure. Purification of the crude product was performed using flash chromatography on silica gel (hexanes $/$ EtOAc $=10: 1-5: 1)$ to afford the corresponding acid S14 (51 $\mathrm{mg}, 90 \%)$ as a colorless oil. TLC: $\mathrm{R}_{f}=0.4$ (hexanes $/$ EtOAc $=3: 1$ ), PMA stain. $\alpha_{\mathrm{D}}^{24}=+20.6$ (c $\left.0.01, \mathrm{CHCl}_{3}\right)$. ${ }^{1} \mathrm{H}$ NMR $\delta 3.29$ (dd, $\left.J=10.3,1.8 \mathrm{~Hz}, 1 \mathrm{H}\right), 2.52-2.36(\mathrm{~m}, 1 \mathrm{H}), 1.77-1.64(\mathrm{~m}, 1 \mathrm{H}), 1.67-1.57$ $(\mathrm{m}, 1 \mathrm{H}), 1.49-1.37(\mathrm{~m}, 2 \mathrm{H}), 1.40-1.27(\mathrm{~m}, 4 \mathrm{H}), 1.25-1.13(\mathrm{~m}, 2 \mathrm{H}), 1.16(\mathrm{~d}, \mathrm{~J}=7.0 \mathrm{~Hz}, 3 \mathrm{H})$, $1.08-0.95(\mathrm{~m}, 1 \mathrm{H}), 0.91(\mathrm{~d}, J=6.7 \mathrm{~Hz}, 3 \mathrm{H}), 0.87(\mathrm{~s}, 9 \mathrm{H}) .{ }^{13} \mathrm{C} \mathrm{NMR}\left(100 \mathrm{MHz}, \mathrm{CDCl}_{3}\right) \delta 182.7$, 77.8, 39.5, 39.3, 35.2, 35.0, 33.6, 29.8, 27.5, 26.7, 25.7, 21.1, 17.1. HRMS (ESI) calculated for $\mathrm{C}_{15} \mathrm{H}_{30} \mathrm{O}_{3} \mathrm{Na}^{+}[\mathrm{M}+\mathrm{Na}]^{+}$281.2087, found 281.2091.

\subsubsection{Synthesis of (2S,7R,9R)-N-allyl-9-hydroxy-N,2,7,10,10-pentamethylundecanamide (18)}

To a solution of acid S14 (39 mg, $0.15 \mathrm{mmol}, 1.0$ eq.) and $N$-allylmethylamine $(29 \mu \mathrm{L}$, $0.30 \mathrm{mmol}, 2.0$ eq.) in dry DCM ( $2 \mathrm{~mL}, 0.08 \mathrm{M})$, HOAt (41 mg, $0.30 \mathrm{mmol}, 2.0$ eq.), DMAP (56 mg, $0.46 \mathrm{mmol}, 3.0$ eq.) and EDCI (58 mg, $0.30 \mathrm{mmol}, 2.0$ eq.) were sequentially added at $0{ }^{\circ} \mathrm{C}$. The reaction mixture was allowed to stir at room temperature for $12 \mathrm{~h}$, quenched with saturated aqueous solution of $\mathrm{NaHCO}_{3}(5 \mathrm{~mL})$ and extracted with $\mathrm{DCM}(3 \times 5 \mathrm{~mL})$. The combined organic layers were washed with brine $(5 \mathrm{~mL})$, dried over anhydrous sodium sulfate, filtered and concentrated under reduced pressure. Purification of the crude product was performed using flash chromatography on silica gel (hexanes/EtOAc $=3: 1$ ) to afford the amide 18 (32 mg, 68\%) as a colorless oil. TLC: $\mathrm{R}_{f}=0.4$ (hexanes $/$ EtOAc $=2: 1$ ), iodine and PMA stain. $\alpha_{\mathrm{D}}^{26}=+26.0\left(c 0.01, \mathrm{CHCl}_{3}\right) .{ }^{1} \mathrm{H} \mathrm{NMR}\left(400 \mathrm{MHz}, \mathrm{CDCl}_{3}\right.$ as a 1:1 mixture of two major conformers) $\delta 5.82-5.66(\mathrm{~m}, 1 \mathrm{H}), 5.24-5.04(\mathrm{~m}, 2 \mathrm{H}), 4.07-3.94(\mathrm{~m}, 1 \mathrm{H}), 3.94-3.90$ $(\mathrm{m}, 1 \mathrm{H}), 3.34-3.20(\mathrm{~m}, 1 \mathrm{H}),(2.97$ and $2.91(\mathrm{~s}), 3 \mathrm{H}), 2.74-2.52(\mathrm{~m}, 1 \mathrm{H}), 1.87(\mathrm{~s}, 1 \mathrm{H}), 1.78-1.66$ (m, 1H), 1.65-1.55 (m, 1H), 1.51-1.40 (m, 1H), 1.36-1.32 (m, 1H), 1.32-1.29 (m, 1H), 1.29$1.26(\mathrm{~m}, 1 \mathrm{H}), 1.26-1.21(\mathrm{~m}, 2 \mathrm{H}), 1.21-1.19(\mathrm{~m}, 1 \mathrm{H}), 1.18-1.12(\mathrm{~m}, 1 \mathrm{H}),(1.09$ and $1.08(\mathrm{~d}$, $J=6.7 \mathrm{~Hz}), 3 \mathrm{H}), 1.04-0.92(\mathrm{~m}, 1 \mathrm{H}),(0.89$ and $0.89(\mathrm{~d}, J=6.7 \mathrm{~Hz}), 3 \mathrm{H}), 0.87(\mathrm{~s}, 9 \mathrm{H}) .{ }^{13} \mathrm{C}$ NMR $\left(100 \mathrm{MHz}, \mathrm{CDCl}_{3}\right.$ as a $1: 1$ mixture of two major conformers) $\delta 177.3$ and 176.6, 133.4 and 133.1, 117.0 and $116.6,77.4,52.2$ and 50.3, 39.5 and $39.5,36.0$ and $35.8,35.1$ and $35.0,34.9$ and 34.8, 34.4, 34.1 and 34.0, 29.5 and 29.5, 27.7 and 27.7, 26.8 and 26.8, 25.8 and 25.8, 21.2 and 21.2, 18.4 and 17.8. HRMS (ESI) calculated for $\mathrm{C}_{19} \mathrm{H}_{37} \mathrm{NO}_{2} \mathrm{Na}^{+}[\mathrm{M}+\mathrm{Na}]^{+} 334.2717$, found 334.2717 .

3.2.14. Synthesis of (3R,5R,10S)-11-(allyl(methyl)amino)-2,2,5,10-tetramethyl-11-oxoundecan-3-yl acrylate (19)

To a solution of alcohol 18 (32 mg, $0.1 \mathrm{mmol}, 1.0$ eq.) in dry DCM (2 mL, $0.05 \mathrm{M})$, $\mathrm{Et}_{3} \mathrm{~N}(69 \mu \mathrm{L}, 0.5 \mathrm{mmol}, 5.0$ eq.), DMAP (2.4 mg, $0.02 \mathrm{mmol}, 0.2 \mathrm{eq}$.) and acryloyl chloride $\left(24 \mu \mathrm{L}, 0.3 \mathrm{mmol}, 3.0\right.$ eq.) were sequentially added at $0{ }^{\circ} \mathrm{C}$. The reaction mixture was allowed to stir at room temperature for $12 \mathrm{~h}$, quenched with water $(1 \mathrm{~mL})$ and extracted 
with DCM $(3 \times 3 \mathrm{~mL})$. The combined organic layers were washed with brine $(2 \mathrm{~mL})$, dried over anhydrous sodium sulfate, filtered and concentrated under reduced pressure. Purification of the crude product was performed using flash chromatography on silica gel (hexanes $/$ EtOAc $=5: 1)$ to afford ester $19(33 \mathrm{mg}, 90 \%)$ as a colorless oil. TLC: $\mathrm{R}_{f}=0.4$ (hexanes $/$ EtOAc $=3: 1)$, iodine and PMA stain. $\alpha_{\mathrm{D}}^{26}=+23.6\left(c 0.01, \mathrm{CHCl}_{3}\right) .{ }^{1} \mathrm{H}$ NMR $\left(400 \mathrm{MHz}, \mathrm{CDCl}_{3}\right.$ as a $1: 1$ mixture of two major conformers) $\delta 6.37(\mathrm{dd}, J=17.3,1.6 \mathrm{~Hz}, 1 \mathrm{H})$, $6.11(\mathrm{dd}, J=17.3,10.4 \mathrm{~Hz}, 1 \mathrm{H}), 5.80(\mathrm{dd}, J=10.4,1.6 \mathrm{~Hz}, 1 \mathrm{H}), 5.80-5.66(\mathrm{~m}, 1 \mathrm{H}), 5.26-5.04$ $(\mathrm{m}, 2 \mathrm{H}), 4.88(\mathrm{ddd}, J=7.9,4.3,1.1 \mathrm{~Hz}, 1 \mathrm{H}), 4.06-3.95(\mathrm{~m}, 1 \mathrm{H}), 3.98-3.89(\mathrm{~m}, 1 \mathrm{H}),(2.97$ and $2.92(\mathrm{~s}, 3 \mathrm{H})), 2.75-2.52(\mathrm{~m}, 1 \mathrm{H}), 1.71-1.58(\mathrm{~m}, 1 \mathrm{H}), 1.51-1.36(\mathrm{~m}, 3 \mathrm{H}), 1.37-1.23(\mathrm{~m}, 3 \mathrm{H})$, $1.27-1.10(\mathrm{~m}, 3 \mathrm{H}),(1.09$ and $1.08(\mathrm{~d}, J=6.8 \mathrm{~Hz}, 3 \mathrm{H})), 1.06-0.94(\mathrm{~m}, 1 \mathrm{H}), 0.88(\mathrm{~s}, 9 \mathrm{H}),(0.85$ and $0.84(\mathrm{~d}, J=6.5 \mathrm{~Hz}, 3 \mathrm{H})) .{ }^{13} \mathrm{C} \mathrm{NMR}\left(100 \mathrm{MHz}, \mathrm{CDCl}_{3}\right.$ as a $1: 1$ mixture of two major conformers) $\delta 177.4$ and 176.7, 166.2, 133.5 and 133.2, 130.3, 129.1, 117.0 and 116.6, 79.2, 52.2 and 50.2, 37.3, 35.9 and 35.7, 35.7, 35.0, 34.8 and 34.7, 34.4 and 34.0, 29.8, 28.1 and 28.1, 27.0 and 27.0, 26.0, 20.9, 18.24 and 17.6. HRMS (ESI) calculated for $\mathrm{C}_{22} \mathrm{H}_{39} \mathrm{NO}_{3} \mathrm{Na}^{+}[\mathrm{M}+\mathrm{Na}]^{+}$ 388.2822 , found 388.2822 .

\subsubsection{Synthesis of (2S,7R,9R)-laingolide A (1a)}

To a solution of diene 19 (33 mg, $0.09 \mathrm{mmol}, 1.0$ eq.) in DCE (90 mL, $0.001 \mathrm{M}$ ) at room temperature, second-generation Grubbs catalyst (G-II) $(7.6 \mathrm{mg}, 0.009 \mathrm{mmol}, 0.1 \mathrm{eq}$.$) was$ added. The reaction mixture was heated at $80{ }^{\circ} \mathrm{C}$ for $24 \mathrm{~h}$ and then a second portion of G-II (7.6 mg, $0.009 \mathrm{mmol}, 0.1$ eq.) was added. The reaction mixture was kept at $80^{\circ} \mathrm{C}$, stirred for another $24 \mathrm{~h}$ and then concentrated under reduced pressure. Purification of the crude product was performed using flash chromatography on silica gel (hexanes/EtOAc $=5: 1$ ) to afford an inseparable mixture of the desired product S15 and a minor unidentified byproduct as a white solid.

To a solution of the above mixture of $\mathbf{S 1 5}$ and a minor unidentified byproduct in degassed dry toluene $(1 \mathrm{~mL})$ under argon, a solution of $\mathrm{RuH}\left(\mathrm{PPh}_{3}\right)_{3}(\mathrm{CO}) \mathrm{Cl}(8.6 \mathrm{mg}$, $0.009 \mathrm{mmol}, 0.1$ eq.) was added in degassed dry toluene $(7 \mathrm{~mL})$. The reaction mixture was heated to reflux for $24 \mathrm{~h}$, cooled to room temperature, then concentrated under reduced pressure to provide the crude product. Purification of the crude product was performed using flash chromatography on silica gel (hexanes $/$ EtOAc $=5: 1$ ) to afford $(2 S, 7 R, 9 R)$ laingolide A (1a) (13.6 mg, 45\% for two steps) as a white solid. TLC: $\mathrm{R}_{f}=0.6$ (hexanes / EtOAc $=2: 1)$, UV and PMA stain. $\alpha_{\mathrm{D}}^{26}=+301(c 0.01, \mathrm{MeOH}) .{ }^{1} \mathrm{H} \mathrm{NMR}\left(400 \mathrm{MHz}, \mathrm{CDCl}_{3}\right)$ $\delta 6.76(\mathrm{~d}, J=13.8 \mathrm{~Hz}, 1 \mathrm{H}), 5.21(\mathrm{ddd}, J=13.8,9.4,6.0 \mathrm{~Hz}, 1 \mathrm{H}), 4.94(\mathrm{dd}, J=11.1,2.5 \mathrm{~Hz}$, $1 \mathrm{H}), 3.17-3.08(\mathrm{~m}, 1 \mathrm{H}), 3.11(\mathrm{~s}, 3 \mathrm{H}), 2.98$ (ddd, $J=16.0,9.5,0.8 \mathrm{~Hz}, 1 \mathrm{H}), 2.86-2.72(\mathrm{~m}, 1 \mathrm{H})$, $1.80-1.63(\mathrm{~m}, 2 \mathrm{H}), 1.59-1.52(\mathrm{~m}, 1 \mathrm{H}), 1.46-1.40(\mathrm{~m}, 1 \mathrm{H}), 1.40-1.33(\mathrm{~m}, 2 \mathrm{H}), 1.32-1.27(\mathrm{~m}, 2 \mathrm{H})$, $1.25-1.17(\mathrm{~m}, 2 \mathrm{H}), 1.15(\mathrm{~d}, J=6.5 \mathrm{~Hz}, 3 \mathrm{H}), 0.96-0.91(\mathrm{~m}, 1 \mathrm{H}), 0.89(\mathrm{~d}, J=6.8 \mathrm{~Hz}, 3 \mathrm{H}), 0.88$ (s, 9H). ${ }^{13} \mathrm{C} \mathrm{NMR}\left(100 \mathrm{MHz}, \mathrm{CDCl}_{3}\right) \delta 176.3,173.3,133.4,104.2,77.9,37.7,37.1,35.7,35.4,34.3$, $34.2,31.3,30.5,27.6,27.4,26.7,20.1,17.2$. HRMS (ESI) calculated for $\mathrm{C}_{20} \mathrm{H}_{35} \mathrm{NO}_{3} \mathrm{Na}^{+}[\mathrm{M}+$ $\mathrm{Na}]^{+} 360.2509$, found 360.2510 .

3.2.16. Synthesis of (6R,11R,13R,Z)-13-(tert-butyl)-2,2,3,3,6,11,15,15,16,16-decamethyl-4,14dioxa-3,15-disilaheptadec-7-ene (20)

Product 20 was synthesised according to the procedures for the synthesis of $\mathbf{1 6}$ from 12 (910 mg, $3.0 \mathrm{mmol}, 1.0$ eq.) and sulfone ent-8 (1.4 g, $3.6 \mathrm{mmol}, 1.2 \mathrm{eq.})$. Purification of the crude product was performed using flash chromatography on silica gel (hexanes $/$ EtOAc $=100: 1)$ to afford $20(998 \mathrm{mg}, 70 \%)$ as a colorless oil. TLC: $\mathrm{R}_{f}=0.6$ (hexanes $/ \mathrm{EtOAc}=80: 1)$, iodine and PMA stain. $\alpha_{\mathrm{D}}^{26}=+1.10\left(c 0.01, \mathrm{CHCl}_{3}\right) .{ }^{1} \mathrm{H} \mathrm{NMR}(400 \mathrm{MHz}$, $\mathrm{CDCl}_{3}$ as a mixture of $\left.\mathrm{Z} / E=3: 1\right) \delta(5.50-5.26(\mathrm{~m})$ and $5.14(\mathrm{ddd}, J=10.9,9.4,1.5 \mathrm{~Hz}) 2 \mathrm{H})$, 3.55-3.42 (m, 1H), 3.41-3.31 (m, 1H), 3.34-3.27 (m, 1H), (2.78-2.54 $(\mathrm{m})$ and 2.37-2.20 (m), $1 \mathrm{H}), 2.22-2.03(\mathrm{~m}, 1 \mathrm{H}), 2.07-1.94(\mathrm{~m}, 1 \mathrm{H}), 1.69-1.51(\mathrm{~m}, 1 \mathrm{H}), 1.51-1.37(\mathrm{~m}, 2 \mathrm{H}), 1.26-1.13(\mathrm{~m}$, $1 \mathrm{H}), 1.10-0.98(\mathrm{~m}, 1 \mathrm{H}),(0.96(\mathrm{~d}, J=6.7 \mathrm{~Hz})$ and $0.96(\mathrm{~d}, J=6.7 \mathrm{~Hz}), 3 \mathrm{H}), 0.95-0.86(\mathrm{~m}, 21 \mathrm{H})$, 0.88-0.83 (m, 9H), 0.07-0.03 (m, 12H). $\left.{ }^{13} \mathrm{C} \mathrm{NMR} \mathrm{(100} \mathrm{MHz,} \mathrm{CDCl}_{3}\right) \delta 132.8,132.6,130.5$, 
$130.4,78.7,78.6,68.5,68.2,41.9,39.5,36.7,36.4,36.0,36.0,35.0,30.3,30.0,29.8,26.6,26.4$, 26.2, 26.2, 25.2, 20.9, 18.7, 18.7, 18.6, 18.5, 17.7, 16.9, -3.2, -3.2, -3.7, -5.1, -5.1. HRMS (ESI) calculated for $\mathrm{C}_{27} \mathrm{H}_{58} \mathrm{O}_{2} \mathrm{Si}_{2} \mathrm{Na}^{+}[\mathrm{M}+\mathrm{Na}]^{+} 493.3869$, found 493.3866 .

\subsubsection{Synthesis of (2R,7R,9R)-2,7,10,10-tetramethylundecane-1,9-diol (21)}

Product $\mathbf{2 1}$ was synthesised according to the procedures for the synthesis of $\mathbf{1 7}$ from 20 (250 mg, $0.53 \mathrm{mmol}, 1.0$ eq.). Purification of the crude product was performed using flash chromatography on silica gel (hexanes/EtOAc $=3: 1)$ to afford $21(59.5 \mathrm{mg}, 46 \%)$ as a colorless oil. TLC: $\mathrm{R}_{f}=0.3$ (hexanes $/$ EtOAc $=3: 1$ ), PMA stain. $\alpha_{\mathrm{D}}^{27}=+27.7$ (c $\left.0.01, \mathrm{CHCl}_{3}\right)$. ${ }^{1} \mathrm{H}$ NMR (500 MHz, $\left.\mathrm{CDCl}_{3}\right) \delta 3.47(\mathrm{dd}, J=10.4,5.9 \mathrm{~Hz}, 1 \mathrm{H}), 3.39(\mathrm{dd}, J=10.5,6.5 \mathrm{~Hz}, 1 \mathrm{H})$, $3.26(\mathrm{dd}, J=10.3,1.8 \mathrm{~Hz}, 1 \mathrm{H}), 1.80(\mathrm{~s}, 2 \mathrm{H}), 1.72-1.51(\mathrm{~m}, 2 \mathrm{H}), 1.47-1.35(\mathrm{~m}, 2 \mathrm{H}), 1.37-1.21$ (m, 5H), 1.21-1.06 (m, 2H), $1.01(\mathrm{ddd}, J=18.2,9.5,3.4 \mathrm{~Hz}, 1 \mathrm{H}), 0.91(\mathrm{~d}, J=6.7 \mathrm{~Hz}, 3 \mathrm{H}), 0.89$ $(\mathrm{d}, J=6.7 \mathrm{~Hz}, 3 \mathrm{H}), 0.86$ (s, 9H). ${ }^{13} \mathrm{C} \mathrm{NMR}\left(125 \mathrm{MHz}, \mathrm{CDCl}_{3}\right) \delta 77.7,68.4,39.5,35.8,35.5$, $35.0,33.2,30.0,27.2,27.1,25.8,21.1,16.6$. HRMS (ESI) calculated for $\mathrm{C}_{15} \mathrm{H}_{32} \mathrm{O}_{2} \mathrm{Na}^{+}[\mathrm{M}+$ $\mathrm{Na}]^{+}$267.2295, found 267.2293.

\subsubsection{Synthesis of (2R,7R,9R)-9-hydroxy-2,7,10,10-tetramethylundecanoic acid (S16)}

Product S16 was synthesised according to the procedures for the synthesis of S14 from 21 ( $258 \mathrm{mg}, 1.06 \mathrm{mmol}, 1.0$ eq.). Purification of the crude product was performed using flash chromatography on silica gel (hexanes $/$ EtOAc $=10: 1-5: 1)$ to afford S16 $(246 \mathrm{mg}$, 90\%) as a colorless oil. TLC: $\mathrm{R}_{f}=0.4$ (hexanes $\left./ \mathrm{EtOAc}=3: 1\right)$, PMA stain. $\alpha_{\mathrm{D}}^{27}=+17.7(c 0.01$, $\left.\mathrm{CHCl}_{3}\right) .{ }^{1} \mathrm{H}$ NMR $\left(400 \mathrm{MHz}, \mathrm{CDCl}_{3}\right) \delta 3.28(\mathrm{dd}, J=10.3,1.8 \mathrm{~Hz}, 1 \mathrm{H}), 2.53-2.32(\mathrm{~m}, 1 \mathrm{H})$, $1.82-1.56(\mathrm{~m}, 2 \mathrm{H}), 1.49-1.37(\mathrm{~m}, 2 \mathrm{H}), 1.39-1.17(\mathrm{~m}, 5 \mathrm{H}), 1.21-1.13(\mathrm{~m}, 1 \mathrm{H}), 1.16(\mathrm{~d}, J=6.9 \mathrm{~Hz}$, $3 \mathrm{H}), 1.09-0.95(\mathrm{~m}, 1 \mathrm{H}), 0.91(\mathrm{~d}, J=6.7 \mathrm{~Hz}, 3 \mathrm{H}), 0.87(\mathrm{~s}, 9 \mathrm{H}) .{ }^{13} \mathrm{C} \mathrm{NMR}\left(100 \mathrm{MHz}, \mathrm{CDCl}_{3}\right) \delta$ 182.8, 77.9, 39.5, 39.3, 35.4, 35.0, 33.7, 30.0, 27.6, 26.8, 25.8, 21.1, 16.9. HRMS (ESI) calculated for $\mathrm{C}_{15} \mathrm{H}_{30} \mathrm{O}_{3} \mathrm{Na}^{+}[\mathrm{M}+\mathrm{Na}]^{+} 281.2087$, found 281.2088.

3.2.19. Synthesis of (2R,7R,9R)-N-allyl-9-hydroxy-N,2,7,10,10-pentamethylundecanamide (22)

Product 22 was synthesised according to the procedures for the synthesis of $\mathbf{1 8}$ from S16 (105 mg, $0.4 \mathrm{mmol}, 1.0$ eq.). Purification of the crude product was performed using flash chromatography on silica gel (hexanes/EtOAc $=3: 1)$ to afford $22(83.4 \mathrm{mg}, 67 \%)$ as a colorless oil. TLC: $\mathrm{R}_{f}=0.4$ (hexanes $/$ EtOAc $=2: 1$ ), iodine and PMA stain. $\alpha_{\mathrm{D}}^{27}=+10.6$ (c $\left.0.01, \mathrm{CHCl}_{3}\right) .{ }^{1} \mathrm{H} \mathrm{NMR}\left(400 \mathrm{MHz}, \mathrm{CDCl}_{3}\right.$ as a $1: 1$ mixture of two major conformers) $\delta$ 6.04-5.59 (m, 1H), 5.26-4.94 (m, 2H), 4.14-3.94 (m, 1H), 3.97-3.88 (m, 1H), $3.26(\mathrm{dd}, J=10.2$, $1.7 \mathrm{~Hz}, 1 \mathrm{H}),(2.97$ and $2.92(\mathrm{~s}, 3 \mathrm{H})), 2.79-2.48(\mathrm{~m}, 1 \mathrm{H}), 1.80-1.55(\mathrm{~m}, 3 \mathrm{H}), 1.44-1.28(\mathrm{~m}, 3 \mathrm{H})$, $1.29-1.25(\mathrm{~m}, 1 \mathrm{H}), 1.24(\mathrm{~s}, 1 \mathrm{H}), 1.22-1.12(\mathrm{~m}, 3 \mathrm{H}),(1.10$ and $1.08(\mathrm{~d}, J=6.7 \mathrm{~Hz}, 3 \mathrm{H})), 1.05-0.95$ $(\mathrm{m}, 1 \mathrm{H}),(0.91$ and $0.90(\mathrm{~d}, J=6.8 \mathrm{~Hz}, 3 \mathrm{H})), 0.87(\mathrm{~s}, 9 \mathrm{H}) .{ }^{13} \mathrm{C} \mathrm{NMR}\left(100 \mathrm{MHz}, \mathrm{CDCl}_{3}\right.$ as a $1: 1$ mixture of two major conformers) $\delta 177.3$ and 176.7, 133.4 and 133.2, 117.0 and 116.6, 77.7, 52.1 and 50.2, 39.4, 35.9 and 35.7, 35.6, 35.0, 34.8 and 34.5, 34.2 and 34.0, 30.1 and 30.1, 28.1 and 28.1, 27.1 and 27.0, 25.8 and 25.8, 21.2, 18.2 and 17.6. HRMS (ESI) calculated for $\mathrm{C}_{19} \mathrm{H}_{37} \mathrm{NO}_{2} \mathrm{Na}^{+}[\mathrm{M}+\mathrm{Na}]^{+} 334.2717$, found 334.2718 .

3.2.20. Synthesis of (3R,5R,10R)-11-(allyl(methyl)amino)-2,2,5,10-tetramethylundecan-3-yl acrylate (23)

Product 23 was synthesised according to the procedures for the synthesis of 19 from 22 (63 mg, $0.2 \mathrm{mmol}, 1.0$ eq.). Purification of the crude product was performed using flash chromatography on silica gel (hexanes/EtOAc $=5: 1)$ to afford $23(64.3 \mathrm{mg}, 88 \%)$ as a colorless oil. TLC: $\mathrm{R}_{f}=0.4$ (hexanes $/$ EtOAc $=3: 1$ ), iodine and PMA stain. $\alpha_{\mathrm{D}}^{27}=+8.9$ (c 0.01, $\left.\mathrm{CHCl}_{3}\right) .{ }^{1} \mathrm{H} \mathrm{NMR}\left(400 \mathrm{MHz}, \mathrm{CDCl}_{3}\right.$ as a 1:1 mixture of two major conformers) $\delta 6.37$ (dd, $J=17.3,1.6 \mathrm{~Hz}, 1 \mathrm{H}), 6.11(\mathrm{dd}, J=17.3,10.4 \mathrm{~Hz}, 1 \mathrm{H}), 5.80(\mathrm{dd}, J=10.4,1.6 \mathrm{~Hz}, 1 \mathrm{H}), 5.79-5.66$ $(\mathrm{m}, 1 \mathrm{H}), 5.27-5.04(\mathrm{~m}, 2 \mathrm{H}), 4.94-4.82(\mathrm{~m}, 1 \mathrm{H}), 4.06-3.95(\mathrm{~m}, 1 \mathrm{H}), 3.97-3.84(\mathrm{~m}, 1 \mathrm{H}),(2.97$ and $2.91(\mathrm{~s}, 3 \mathrm{H})), 2.76-2.52(\mathrm{~m}, 1 \mathrm{H}), 1.71-1.58(\mathrm{~m}, 1 \mathrm{H}), 1.41(\mathrm{ddd}, J=7.2,5.6,1.9 \mathrm{~Hz}, 2 \mathrm{H})$, $1.39-1.26(\mathrm{~m}, 1 \mathrm{H}), 1.27-1.16(\mathrm{~m}, 5 \mathrm{H}),(1.10$ and $1.08(\mathrm{~d}, J=6.7 \mathrm{~Hz}, 1 \mathrm{H})), 1.06-0.95(\mathrm{~m}, 1 \mathrm{H})$, $0.88(\mathrm{~s}, 9 \mathrm{H}),(0.85$ and $0.84(\mathrm{~d}, J=6.5 \mathrm{~Hz}, 3 \mathrm{H})) .{ }^{13} \mathrm{C} \mathrm{NMR}\left(100 \mathrm{MHz}, \mathrm{CDCl}_{3}\right.$ as a $1: 1$ mixture 
of two major conformers) $\delta 177.4$ and $176.7,166.3,133.5$ and $133.2,130.3,129.1,117.0$ and 116.6, 79.2, 52.2 and 50.2, 37.2, 35.9 and 35.7, 35.5 and 35.0, 34.8 and 34.6, 34.3, 33.9, 29.7 and 29.7, 28.0 and 28.0, 26.8 and 26.8, 26.0, 20.9, 18.2 and 17.61. HRMS (ESI) calculated for $\mathrm{C}_{22} \mathrm{H}_{39} \mathrm{NO}_{3} \mathrm{Na}^{+}[\mathrm{M}+\mathrm{Na}]^{+}$388.2822, found 388.2822.

\subsubsection{Synthesis of (2R,7R,9R)-laingolide A (1b)}

Product S17 was synthesised according to the procedures for the synthesis of S15 from 23 (10.3 mg, $0.027 \mathrm{mmol}, 1.0$ eq.). Purification of the crude product was performed using flash chromatography on silica gel (hexanes $/$ EtOAc $=5: 1$ ) to afford an inseparable mixture of the desired product $\mathbf{S 1 7}$ and a minor unidentified byproduct $(9 \mathrm{mg})$ as a white solid.

$(2 R, 7 R, 9 R)$-laingolide $\mathrm{A}(\mathbf{1 b})$ was synthesised according to the procedures for the synthesis of $(2 S, 7 S, 9 R)$-laingolide A (1a) from the above mixture of S17 and a minor unidentified byproduct. Purification of the crude product was performed using flash chromatography on silica gel (hexanes/EtOAc $=5: 1)$ to afford $(2 R, 7 R, 9 R)$-laingolide $\mathrm{A}(\mathbf{1 b})$ ( $4 \mathrm{mg}, 44 \%$ for two steps) as a white solid. TLC: $\mathrm{R}_{f}=0.6$ (hexanes $/$ EtOAc $=2: 1$ ), UV and PMA stain. $\alpha_{\mathrm{D}}^{23}=-76.3\left(c 0.005, \mathrm{CHCl}_{3}\right) .{ }^{1} \mathrm{H} \mathrm{NMR}\left(500 \mathrm{MHz}, \mathrm{CDCl}_{3}\right) \delta 7.09(\mathrm{~d}, J=13.9 \mathrm{~Hz}$, $1 \mathrm{H}), 5.16(\mathrm{dt}, J=14.1,7.2 \mathrm{~Hz}, 1 \mathrm{H}), 4.92(\mathrm{dd}, J=11.9,1.8 \mathrm{~Hz}, 1 \mathrm{H}), 3.09$ (s, 3H), 3.05 (ddd, $J=13.3,7.4,0.9 \mathrm{~Hz}, 1 \mathrm{H}), 2.99(\mathrm{ddd}, J=13.3,6.9,1.4 \mathrm{~Hz}, 1 \mathrm{H}), 2.91(\mathrm{dt}, J=13.4,6.5 \mathrm{~Hz}$, $1 \mathrm{H}), 1.65-1.61(\mathrm{~m}, 0 \mathrm{H}), 1.58-1.51(\mathrm{~m}, 2 \mathrm{H}), 1.46-1.36(\mathrm{~m}, 1 \mathrm{H}), 1.33-1.25(\mathrm{~m}, 3 \mathrm{H}), 1.25-$ $1.18(\mathrm{~m}, 2 \mathrm{H}), 1.15(\mathrm{~d}, J=6.6 \mathrm{~Hz}, 3 \mathrm{H}), 1.10-0.99(\mathrm{~m}, 1 \mathrm{H}), 0.88(\mathrm{~s}, 9 \mathrm{H}), 0.85-0.79(\mathrm{~m}, 1 \mathrm{H})$, $0.78(\mathrm{~d}, J=6.4 \mathrm{~Hz}, 3 \mathrm{H}) .{ }^{13} \mathrm{C}$ NMR $\left(125 \mathrm{MHz}, \mathrm{CDCl}_{3}\right) \delta 176.5,172.1,133.8,103.6,78.5,38.6$, 37.3, 35.7, 35.4, 35.3, 34.1, 31.4, 26.7, 26.7, 26.6, 26.1, 20.9, 18.0. HRMS (ESI) calculated for $\mathrm{C}_{20} \mathrm{H}_{35} \mathrm{NO}_{3} \mathrm{Na}^{+}[\mathrm{M}+\mathrm{Na}]^{+}$360.2509, found 360.2510.

3.2.22. Synthesis of (6S,11S,13R,Z)-13-(tert-butyl)-2,2,3,3,6,11,15,15,16,16-decamethyl-4,14dioxa-3,15-disilaheptadec-7-ene (24)

Product $\mathbf{2 4}$ was synthesised according to the procedures for the synthesis of $\mathbf{1 6}$ from 15 (780 mg, $2.6 \mathrm{mmol}, 1.0$ eq.) and sulfone 8 (1.2 g, $3.1 \mathrm{mmol}, 1.2$ eq.). Purification of the crude product was performed using flash chromatography on silica gel (hexanes $/$ EtOAc $=100: 1$ ) to afford $24(831 \mathrm{mg}, 68 \%)$ as a colorless oil. TLC: $\mathrm{R}_{f}=0.6$ (hexanes $/$ EtOAc $\left.=80: 1\right)$, iodine and PMA stain. $\alpha_{\mathrm{D}}^{27}=+18.8\left(c 0.01, \mathrm{CHCl}_{3}\right) .{ }^{1} \mathrm{H} \mathrm{NMR}\left(400 \mathrm{MHz}, \mathrm{CDCl}_{3}\right.$ as a mixture of $Z / E=7: 1) \delta(5.45-5.28(\mathrm{~m})$ and $5.12(\mathrm{ddt}, J=10.9,9.4,1.6 \mathrm{~Hz}), 2 \mathrm{H}), 3.46(\mathrm{dd}, J=9.7,5.9 \mathrm{~Hz}$ $1 \mathrm{H}), 3.35(\mathrm{dd}, J=9.8,7.4 \mathrm{~Hz}, 1 \mathrm{H}), 3.31(\mathrm{dd}, J=8.4,1.7 \mathrm{~Hz}, 2 \mathrm{H}),(2.72-2.57(\mathrm{~m})$ and $2.33-2.21$ (m), 1H), 2.17-1.96 (m, 2H), 1.68-1.54 (m, 1H), 1.41-1.25 (m, 2H), 1.29-1.15 (m, 2H), $0.96(\mathrm{~d}$, $J=6.8 \mathrm{~Hz}, 3 \mathrm{H}), 0.91(\mathrm{~s}, 18 \mathrm{H}), 0.85(\mathrm{~d}, J=6.2 \mathrm{~Hz}, 12 \mathrm{H}), 0.05(\mathrm{~s}, 12 \mathrm{H}) .{ }^{13} \mathrm{C} \mathrm{NMR}(100 \mathrm{MHz}$, $\left.\mathrm{CDCl}_{3}\right) \delta 132.5,130.6,78.7,68.2,41.2,39.0,35.8,35.0,29.4,26.6,26.5,26.2,25.4,19.1,17.7$, $-3.1,-3.5,-5.1,-5.1$. HRMS (ESI) calculated for $\mathrm{C}_{27} \mathrm{H}_{58} \mathrm{O}_{2} \mathrm{Si}_{2} \mathrm{Na}^{+}[\mathrm{M}+\mathrm{Na}]^{+} 493.3869$, found 493.3868 .

\subsubsection{Synthesis of (2S,7S,9R)-2,7,10,10-tetramethylundecane-1,9-diol (25)}

Product $\mathbf{2 5}$ was synthesised according to the procedures for the synthesis of $\mathbf{1 7}$ from 24 (235 mg, $0.5 \mathrm{mmol}, 1.0$ eq.). Purification of the crude product was performed using flash chromatography on silica gel (hexanes/EtOAc $=3: 1)$ to afford $25(55 \mathrm{mg}, 45 \%)$ as a colorless oil. TLC: $\mathrm{R}_{f}=0.3$ (hexanes/EtOAc $\left.=3: 1\right)$, PMA stain. $\alpha_{\mathrm{D}}^{25}=+14.4\left(c 0.01, \mathrm{CHCl}_{3}\right)$. ${ }^{1} \mathrm{H}$ NMR $\left(400 \mathrm{MHz}, \mathrm{CDCl}_{3}\right) \delta 3.48(\mathrm{dd}, J=10.6,5.8 \mathrm{~Hz}, 1 \mathrm{H}), 3.39$ (ddd, $J=10.3,6.5,0.8 \mathrm{~Hz}$, $1 \mathrm{H}), 3.27(\mathrm{dd}, J=10.6,1.8 \mathrm{~Hz}, 1 \mathrm{H}), 1.74-1.53(\mathrm{~m}, 4 \mathrm{H}), 1.46-1.33(\mathrm{~m}, 2 \mathrm{H}), 1.32-1.25(\mathrm{~m}, 4 \mathrm{H})$, 1.24-1.08 (m, 4H), $0.89(\mathrm{~d}, J=6.7 \mathrm{~Hz}, 3 \mathrm{H}), 0.86(\mathrm{~d}, J=7.1 \mathrm{~Hz}, 3 \mathrm{H}), 0.87(\mathrm{~s}, 9 \mathrm{H}) .{ }^{13} \mathrm{C} \mathrm{NMR}$ $\left(100 \mathrm{MHz}_{\mathrm{CDCl}}\right) \delta$ 77.4, 68.4, 39.1, 38.5, 35.8, 34.9, 33.2, 29.7, 27.4, 27.3, 25.8, 19.0, 16.7. HRMS (ESI) calculated for $\mathrm{C}_{15} \mathrm{H}_{32} \mathrm{O}_{2} \mathrm{Na}^{+}[\mathrm{M}+\mathrm{Na}]^{+}$267.2295, found 267.2293. 
3.2.24. Synthesis of (2S,7S,9R)-9-hydroxy-2,7,10,10-tetramethylundecanoic acid (S18)

Product S18 was synthesised according to the procedures for the synthesis of S14 from 25 (50 mg, $0.21 \mathrm{mmol}, 1.0$ eq.). Purification of the crude product was performed using flash chromatography on silica gel (hexanes/EtOAc $=10: 1-5: 1)$ to afford S18 $(47.8 \mathrm{mg}, 91 \%)$ as a colorless oil. TLC: $\mathrm{R}_{f}=0.4$ (hexanes $/$ EtOAc $\left.=3: 1\right)$, PMA stain. $\alpha_{\mathrm{D}}^{27}=+13.8\left(c 0.01, \mathrm{CHCl}_{3}\right)$. ${ }^{1} \mathrm{H}$ NMR $\left(500 \mathrm{MHz}, \mathrm{CDCl}_{3}\right) \delta 3.29(\mathrm{dd}, J=10.6,1.7 \mathrm{~Hz}, 1 \mathrm{H}), 2.52-2.35(\mathrm{~m}, 1 \mathrm{H}), 1.72-1.64(\mathrm{~m}$, $1 \mathrm{H}), 1.64-1.56(\mathrm{~m}, 1 \mathrm{H}), 1.47-1.39(\mathrm{~m}, 1 \mathrm{H}), 1.35-1.28(\mathrm{~m}, 5 \mathrm{H}), 1.26-1.23(\mathrm{~m}, 1 \mathrm{H}), 1.23-1.10$ $(\mathrm{m}, 2 \mathrm{H}), 1.16(\mathrm{~d}, J=7.0 \mathrm{~Hz}, 3 \mathrm{H}), 0.87(\mathrm{~s}, 9 \mathrm{H}), 0.86(\mathrm{~d}, J=6.6 \mathrm{~Hz}, 3 \mathrm{H}) .{ }^{13} \mathrm{C}$ NMR $(125 \mathrm{MHz}$, $\mathrm{CDCl}_{3}$ ) $\delta 182.6,77.6,39.5,38.9,38.3,34.9,33.7,29.5,27.4,27.0,25.8,19.1,17.0$. HRMS (ESI) calculated for $\mathrm{C}_{15} \mathrm{H}_{30} \mathrm{O}_{3} \mathrm{Na}^{+}[\mathrm{M}+\mathrm{Na}]^{+}$281.2087, found 281.2087.

3.2.25. Synthesis of (2S,7S,9R)-N-allyl-9-hydroxy-N,2,7,10,10-pentamethylundecanamide (26)

Product $\mathbf{2 6}$ was synthesised according to the procedures for the synthesis of $\mathbf{1 8}$ from S18 ( $47.8 \mathrm{mg}, 0.182 \mathrm{mmol}, 1.0$ eq.). Purification of the crude product was performed using flash chromatography on silica gel (hexanes/EtOAc $=3: 1)$ to afford $26(42.5 \mathrm{mg}, 75 \%)$ as a colorless oil. TLC: $\mathrm{R}_{f}=0.4$ (hexanes $/ \mathrm{EtOAc}=2: 1$ ), iodine and PMA stain. $\alpha_{\mathrm{D}}^{25}=+16.7$ (c $\left.0.01, \mathrm{CHCl}_{3}\right) .{ }^{1} \mathrm{H}$ NMR $\left(500 \mathrm{MHz}, \mathrm{CDCl}_{3}\right.$ as a $1: 1$ mixture of two major conformers) $\delta$ $5.82-5.65(\mathrm{~m}, 1 \mathrm{H}), 5.30-4.98(\mathrm{~m}, 2 \mathrm{H}), 4.07-3.92(\mathrm{~m}, 1 \mathrm{H}), 3.91(\mathrm{~d}, J=4.7 \mathrm{~Hz}, 1 \mathrm{H}), 3.26(\mathrm{~d}$, $J=10.5 \mathrm{~Hz}, 1 \mathrm{H}),(2.96$ and $2.90(\mathrm{~s}, 3 \mathrm{H})), 2.73-2.51(\mathrm{~m}, 1 \mathrm{H}), 1.74-1.62(\mathrm{~m}, 1 \mathrm{H}), 1.61-1.55(\mathrm{~m}$, $1 \mathrm{H}), 1.40-1.27(\mathrm{~m}, 3 \mathrm{H}), 1.27-1.18(\mathrm{~m}, 5 \mathrm{H}), 1.17-1.10(\mathrm{~m}, 2 \mathrm{H}),(1.09$ and $1.07(\mathrm{~d}, J=6.9 \mathrm{~Hz}$, $3 \mathrm{H})), 0.86(\mathrm{~s}, 9 \mathrm{H}), 0.84(\mathrm{~d}, J=7.1 \mathrm{~Hz}, 3 \mathrm{H}) .{ }^{13} \mathrm{C} \mathrm{NMR}\left(125 \mathrm{MHz}, \mathrm{CDCl}_{3}\right.$ as a $1: 1$ mixture of two major conformers) $\delta 177.3$ and 176.6, 133.4 and 133.2, 117.0 and 116.6, 77.3, 52.1 and 50.2, 39.0, 38.4, 35.9 and 35.7, 34.9 and 34.8, 34.5 and 34.2, 33.9, 29.7, 28.0 and 27.9, 27.3, 25.8, 19.0, 18.2 and 17.61. HRMS (ESI) calculated for $\mathrm{C}_{19} \mathrm{H}_{37} \mathrm{NO}_{2} \mathrm{Na}^{+}[\mathrm{M}+\mathrm{Na}]^{+} 334.2717$, found 334.2721 .

3.2.26. Synthesis of (3R,5S,10S)-11-(allyl(methyl)amino)-2,2,5,10-tetramethyl-11-oxoundecan-3-yl acrylate (27)

Product 27 was synthesised according to the procedures for the synthesis of $\mathbf{1 9}$ from 26 (116 mg, $0.372 \mathrm{mmol}, 1.0$ eq.). Purification of the crude product was performed using flash chromatography on silica gel (hexanes/EtOAc $=5: 1)$ to afford $27(115 \mathrm{mg}, 85 \%)$ as a colorless oil. TLC: $\mathrm{R}_{f}=0.4$ (hexanes $\left./ \mathrm{EtOAc}=3: 1\right)$, iodine and PMA stain. $\alpha_{\mathrm{D}}^{26}=+8.8(c 0.01$, $\left.\mathrm{CHCl}_{3}\right) .{ }^{1} \mathrm{H} \mathrm{NMR}\left(400 \mathrm{MHz}, \mathrm{CDCl}_{3}\right.$ as a 1:1 mixture of two major conformers) $\delta 6.37$ (dd, $J=17.4,1.5 \mathrm{~Hz}, 1 \mathrm{H}), 6.11(\mathrm{dd}, J=17.3,10.4 \mathrm{~Hz}, 1 \mathrm{H}), 5.80(\mathrm{dd}, J=10.4,1.7 \mathrm{~Hz}, 1 \mathrm{H}), 5.72(\mathrm{ddt}$, $J=16.5,11.5,5.9 \mathrm{~Hz}, 1 \mathrm{H}), 5.24-5.02(\mathrm{~m}, 2 \mathrm{H}), 4.89(\mathrm{~d}, J=10.9 \mathrm{~Hz}, 1 \mathrm{H}), 4.08-3.93(\mathrm{~m}, 1 \mathrm{H})$, 3.95-3.87 (m, 1H), $(2.95$ and $2.91(\mathrm{~s}, 3 \mathrm{H})), 2.72-2.46(\mathrm{~m}, 1 \mathrm{H}), 1.74-1.1(\mathrm{~m}, 1 \mathrm{H}), 1.58-1.52(\mathrm{~m}$, $1 \mathrm{H}), 1.36-1.29(\mathrm{~m}, 1 \mathrm{H}), 1.27-1.12(\mathrm{~m}, 8 \mathrm{H}),(1.08$ and $1.06(\mathrm{~d}, J=6.5 \mathrm{~Hz}, 3 \mathrm{H})), 0.86(\mathrm{~s}, 12 \mathrm{H})$. ${ }^{13} \mathrm{C}$ NMR $\left(100 \mathrm{MHz}, \mathrm{CDCl}_{3}\right.$ as a 1:1 mixture of two major conformers) $\delta 177.2$ and 176.6, $166.3,133.4$ and 133.1, 130.4, 129.0, 117 and 116.6, 79.0, 52.1 and 50.2, 38.1 and 37.1, 35.9 and $35.7,34.8$ and $34.8,34.5,34.2,33.9,29.5,27.9$ and $27.9,27.2,26.1,19.3,18.2$ and 17.6. HRMS (ESI) calculated for $\mathrm{C}_{22} \mathrm{H}_{39} \mathrm{NO}_{3} \mathrm{Na}^{+}[\mathrm{M}+\mathrm{Na}]^{+} 388.2822$, found 388.2824.

\subsubsection{Synthesis of (2S,7S,9R)-laingolide A (1c)}

Product S19 was synthesised according to the procedures for the synthesis of S15 from 27 (28 mg, $0.077 \mathrm{mmol}, 1.0$ eq.). Purification of the crude product was performed using flash chromatography on silica gel (hexanes/EtOAc $=5: 1$ ) to afford an inseparable mixture of the desired product $\mathbf{S 1 9}$ and a minor unidentified byproduct $(20 \mathrm{mg})$ as a white solid.

$(2 S, 7 S, 9 R)$-laingolide A (1c) was synthesised according to the procedures for the synthesis of $(2 S, 7 R, 9 R)$-laingolide A (1a) from the above mixture of $\mathbf{S 1 9}$ and a minor unidentified byproduct. Purification of the crude product was performed using flash chromatography on silica gel (hexanes $/$ EtOAc $=5: 1)$ to afford $(2 S, 7 S, 9 R)$-laingolide A (1c) (13 mg, 50\% for two steps) as a white solid. TLC: $\mathrm{R}_{f}=0.6$ (hexanes $/$ EtOAc $=2: 1$ ), $\mathrm{UV}$ and PMA stain. $\alpha_{\mathrm{D}}^{26}=+145.6\left(c 0.01, \mathrm{CHCl}_{3}\right) .{ }^{1} \mathrm{H}$ NMR $\left(500 \mathrm{MHz}, \mathrm{CDCl}_{3}\right) \delta 7.02(\mathrm{dd}, J=13.7$, 
$1.4 \mathrm{~Hz}, 1 \mathrm{H}), 5.18(\mathrm{ddd}, J=13.6,10.3,5.7 \mathrm{~Hz}, 1 \mathrm{H}), 4.81(\mathrm{dd}, J=11.3,1.3 \mathrm{~Hz}, 1 \mathrm{H}), 3.10$ (s, $3 \mathrm{H}), 3.07(\mathrm{ddd}, J=12.3,5.6,1.5 \mathrm{~Hz}, 1 \mathrm{H}), 3.03-2.95(\mathrm{~m}, 1 \mathrm{H}), 2.94(\mathrm{dd}, J=12.3,10.4 \mathrm{~Hz}, 1 \mathrm{H})$, $1.64-1.57(\mathrm{~m}, 1 \mathrm{H}), 1.60-1.52(\mathrm{~m}, 1 \mathrm{H}), 1.47-1.34(\mathrm{~m}, 1 \mathrm{H}), 1.35-1.27(\mathrm{~m}, 1 \mathrm{H}), 1.30-1.17(\mathrm{~m}, 4 \mathrm{H})$, $1.15(\mathrm{~d}, J=6.5 \mathrm{~Hz}, 3 \mathrm{H}), 1.17-1.12(\mathrm{~m}, 2 \mathrm{H}), 1.03-0.95(\mathrm{~m}, 1 \mathrm{H}), 0.89(\mathrm{~s}, 9 \mathrm{H}), 0.84(\mathrm{~d}, J=5.7 \mathrm{~Hz}$, 3H). ${ }^{13} \mathrm{C} \mathrm{NMR}\left(125 \mathrm{MHz}, \mathrm{CDCl}_{3}\right) \delta$ 176.4, 172.5, 133.5, 105.0, 79.7, 37.6, 36.8, 36.6, 36.2, 35.5, $35.1,31.2,27.7,26.8,26.7,26.2,21.2,18.5$. HRMS (ESI) calculated for $\mathrm{C}_{20} \mathrm{H}_{35} \mathrm{NO}_{3} \mathrm{Na}^{+}[\mathrm{M}+$ $\mathrm{Na}]^{+}$360.2509, found 360.2512.

3.2.28. Synthesis of (6R,11S,13R,Z)-13-(tert-butyl)-2,2,3,3,6,11,15,15,16,16-decamethyl-4,14dioxa-3,15-disilaheptadec-7-ene (28)

Product 28 was synthesised according to the procedures for the synthesis of $\mathbf{1 6}$ from 15 (700 mg, $2.34 \mathrm{mmol}, 1.0$ eq.) and sulfone ent-8 (1.1 g, $2.8 \mathrm{mmol}, 1.2$ eq.). Purification of the crude product was performed using flash chromatography on silica gel (hexanes $/ \mathrm{EtOAc}=100: 1)$ to afford $28(847 \mathrm{mg}, 77 \%)$ as a colorless oil. TLC: $\mathrm{R}_{f}=0.6$ (hexanes $/$ EtOAc $=80: 1)$, iodine and PMA stain. $\alpha_{\mathrm{D}}^{27}=-1.9\left(c 0.01, \mathrm{CHCl}_{3}\right) .{ }^{1} \mathrm{H} \mathrm{NMR}(400 \mathrm{MHz}$, $\mathrm{CDCl}_{3}$ as a mixture of $\left.\mathrm{Z} / E=7: 1\right) \delta(5.50-5.26(\mathrm{~m}), 5.11(\mathrm{ddt}, J=11.0,9.6,1.6 \mathrm{~Hz}), 2 \mathrm{H})$, 3.52-3.43 (m, 1H), 3.39-3.33 (m, 1H), 3.32-3.26 (m, 1H), (2.69-2.55 (m) and 2.32-2.20 (m), $1 \mathrm{H}), 2.14-1.90(\mathrm{~m}, 2 \mathrm{H}), 1.69-1.49(\mathrm{~m}, 1 \mathrm{H}), 1.38-1.32(\mathrm{~m}, 1 \mathrm{H}), 1.31-1.24(\mathrm{~m}, 1 \mathrm{H}), 1.26-1.12(\mathrm{~m}$, $2 \mathrm{H}),(0.97(\mathrm{~d}, J=6.8 \mathrm{~Hz})$ and $0.95(\mathrm{~d}, J=6.7 \mathrm{~Hz}), 3 \mathrm{H}), 0.90(\mathrm{~s}, 18 \mathrm{H}), 0.87-0.81(\mathrm{~m}, 12 \mathrm{H}), 0.04$ $(\mathrm{m}, 12 \mathrm{H}) .{ }^{13} \mathrm{C} \mathrm{NMR}\left(100 \mathrm{MHz}, \mathrm{CDCl}_{3}\right) \delta 132.5,130.5,78.6,68.2,41.1,39.0,35.8,35.0,29.3$, 26.6, 26.4, 26.1, 25.4, 19.2, 18.7, 18.5, 17.7, -3.1, -3.5, -5.1, -5.1. HRMS (ESI) calculated for $\mathrm{C}_{27} \mathrm{H}_{58} \mathrm{O}_{2} \mathrm{Si}_{2} \mathrm{Na}^{+}[\mathrm{M}+\mathrm{Na}]^{+} 493.3869$, found 493.3866 .

\subsubsection{Synthesis of (2R,7S,9R)-2,7,10,10-tetramethylundecane-1,9-diol (29)}

Product 29 was synthesised according to the procedures for the synthesis of $\mathbf{1 7}$ from 28 (250 mg, $0.53 \mathrm{mmol}, 1.0$ eq.). Purification of the crude product was performed using flash chromatography on silica gel (hexanes/EtOAc $=3: 1)$ to afford $29(60.8 \mathrm{mg}, 47 \%)$ as a colorless oil. TLC: $\mathrm{R}_{f}=0.3$ (hexanes $/$ EtOAc $=3: 1$ ), PMA stain. $\alpha_{\mathrm{D}}^{26}=+19.1\left(c 0.01, \mathrm{CHCl}_{3}\right)$. ${ }^{1} \mathrm{H} \mathrm{NMR}\left(400 \mathrm{MHz}, \mathrm{CDCl}_{3}\right) \delta 3.44(\mathrm{ddd}, J=10.2,5.8,1.7 \mathrm{~Hz}, 1 \mathrm{H}), 3.35$ (ddd, $J=10.5,6.5$, $1.9 \mathrm{~Hz}, 1 \mathrm{H}), 3.24(\mathrm{dt}, J=10.6,1.5 \mathrm{~Hz}, 1 \mathrm{H}), 2.14(\mathrm{~d}, J=31.1 \mathrm{~Hz}, 2 \mathrm{H}), 1.68-1.50(\mathrm{~m}, 2 \mathrm{H})$, $1.42-1.30(\mathrm{~m}, 2 \mathrm{H}), 1.30-1.19(\mathrm{~m}, 5 \mathrm{H}), 1.17-0.98(\mathrm{~m}, 3 \mathrm{H}), 0.87(\mathrm{~d}, J=6.7 \mathrm{~Hz}, 3 \mathrm{H}), 0.85-0.82$ (m, 12H). ${ }^{13} \mathrm{C}$ NMR (100 MHz, $\left.\mathrm{CDCl}_{3}\right) \delta 77.3,68.2,39.0,38.4,35.8,34.9,33.2,29.6,27.4$, 27.3, 25.8, 19.0, 16.7. HRMS (ESI) calculated for $\mathrm{C}_{15} \mathrm{H}_{32} \mathrm{O}_{2} \mathrm{Na}^{+}[\mathrm{M}+\mathrm{Na}]^{+}$267.2295, found 267.2295 .

\subsubsection{Synthesis of (2R,7S,9R)-9-hydroxy-2,7,10,10-tetramethylundecanoic acid (S20)}

Product S20 was synthesised according to the procedures for the synthesis of S14 from 29 (112 mg, $0.46 \mathrm{mmol}, 1.0$ eq.). Purification of the crude product was performed using flash chromatography on silica gel (hexanes $/$ EtOAc $=10: 1-5: 1)$ to afford S20 (108 mg, 91\%) as a colorless oil. TLC: $\mathrm{R}_{f}=0.4$ (hexanes $/$ EtOAc $\left.=3: 1\right)$, PMA stain. $\alpha_{\mathrm{D}}^{27}=+8.4(c 0.01$, $\left.\mathrm{CHCl}_{3}\right) .{ }^{1} \mathrm{H}$ NMR $\left(500 \mathrm{MHz}, \mathrm{CDCl}_{3}\right) \delta 3.29(\mathrm{dd}, J=10.6,1.7 \mathrm{~Hz}, 1 \mathrm{H}), 2.51-2.37(\mathrm{~m}, 1 \mathrm{H})$, $1.78-1.57$ (m, 2H), 1.46-1.38 (m, 1H), 1.36-1.28 (m, 5H), 1.28-1.23 (m, 1H), 1.22-1.18 (m, 1H), $1.16(\mathrm{~d}, J=6.9 \mathrm{~Hz}, 3 \mathrm{H}), 1.16-1.13(\mathrm{~m}, 1 \mathrm{H}), 0.88(\mathrm{~s}, 9 \mathrm{H}), 0.86(\mathrm{~d}, J=6.5 \mathrm{~Hz}, 3 \mathrm{H}) .{ }^{13} \mathrm{C}$ NMR $\left(125 \mathrm{MHz}_{2} \mathrm{CDCl}_{3}\right) \delta$ 182.7, 77.6, 39.5, 39.0, 38.2, 34.9, 33.7, 29.6, 27.5, 27.0, 25.8, 19.0, 17.1. HRMS (ESI) calculated for $\mathrm{C}_{15} \mathrm{H}_{30} \mathrm{O}_{3} \mathrm{Na}^{+}[\mathrm{M}+\mathrm{Na}]^{+}$281.2087, found 281.2087.

\subsubsection{Synthesis of (2R,7S,9R)-N-allyl-9-hydroxy-N,2,7,10,10-pentamethylundecanamide (30)}

Product 30 was synthesised according to the procedures for the synthesis of $\mathbf{1 8}$ from S20 (116 mg, $0.45 \mathrm{mmol}, 1.0$ eq.). Purification of the crude product was performed using flash chromatography on silica gel (hexanes/EtOAc = 3:1) to afford $30(105 \mathrm{mg}, 75 \%)$ as a colorless oil. TLC: $\mathrm{R}_{f}=0.4$ (hexanes $/$ EtOAc $=2: 1$ ), iodine and PMA stain. $\alpha_{\mathrm{D}}^{26}=+2.8$ (c $\left.0.01, \mathrm{CHCl}_{3}\right) .{ }^{1} \mathrm{H} \mathrm{NMR}\left(400 \mathrm{MHz}, \mathrm{CDCl}_{3}\right.$ as a $1: 1$ mixture of two major conformers) $\delta$ 5.97-5.56 (m, 1H), 5.30-5.00 (m, 2H), 4.04-3.92 (m, 1H), $3.93(\mathrm{dt}, J=4.9,1.8 \mathrm{~Hz}, 1 \mathrm{H}), 3.27(\mathrm{dt}$, 
$J=10.3,1.4 \mathrm{~Hz}, 1 \mathrm{H}),(2.97$ and $2.92(\mathrm{~s}, 3 \mathrm{H})), 2.79-2.43(\mathrm{~m}, 1 \mathrm{H}), 1.79-1.53(\mathrm{~m}, 3 \mathrm{H}), 1.38-1.23$ $(\mathrm{m}, 6 \mathrm{H}), 1.21-1.13(\mathrm{~m}, 3 \mathrm{H}),(1.10$ and $1.08(\mathrm{~d}, J=6.8 \mathrm{~Hz}, 3 \mathrm{H})), 0.87(\mathrm{~s}, 9 \mathrm{H}),(0.85$ and $0.85(\mathrm{~d}$, $J=6.5 \mathrm{~Hz}, 3 \mathrm{H})) .{ }^{13} \mathrm{C} \mathrm{NMR}\left(100 \mathrm{MHz}, \mathrm{CDCl}_{3}\right.$ as a $1: 1$ mixture of two major conformers $) \delta$ 177.3 and 176.6, 133.4 and 133.2, 117.0 and 116.6, 77.4, 52.2 and 50.2, 39.1, 38.4 and 38.3, 35.9 and 35.7, 34.9 and 34.8, 34.5 and 34.3, 33.9, 29.6, 27.9 and 27.9, 27.3, 25.8, 19.0, 18.3 and 17.7. HRMS (ESI) calculated for $\mathrm{C}_{19} \mathrm{H}_{37} \mathrm{NO}_{2} \mathrm{Na}^{+}[\mathrm{M}+\mathrm{Na}]^{+} 334.2717$, found 334.2716.

3.2.32. Synthesis of (3R,5S,10R)-11-(allyl(methyl)amino)-2,2,5,10-tetramethyl-11-oxoundecan-3-yl acrylate (31)

Product 31 was synthesised according to the procedures for the synthesis of $\mathbf{1 9}$ from 30 (88 mg, $0.28 \mathrm{mmol}, 1.0$ eq.). Purification of the crude product was performed using flash chromatography on silica gel (hexanes/EtOAc $=5: 1)$ to afford $31(88 \mathrm{mg}, 86 \%)$ as a colorless oil. TLC: $\mathrm{R}_{f}=0.4$ (hexanes $/$ EtOAc $=3: 1$ ), iodine and PMA stain. $\alpha_{\mathrm{D}}^{27}=+12.1$ (c $\left.0.01, \mathrm{CHCl}_{3}\right) .{ }^{1} \mathrm{H}$ NMR $\left(400 \mathrm{MHz}, \mathrm{CDCl}_{3}\right.$ as a $1: 1$ mixture of two major conformers) $\delta$ $6.38(\mathrm{dd}, J=17.3,1.6 \mathrm{~Hz}, 1 \mathrm{H}), 6.12(\mathrm{dd}, J=17.3,10.4 \mathrm{~Hz}, 1 \mathrm{H}), 5.80(\mathrm{dd}, J=10.3,1.6 \mathrm{~Hz}$, $1 \mathrm{H}), 5.78-5.66(\mathrm{~m}, 1 \mathrm{H}), 5.33-5.04(\mathrm{~m}, 2 \mathrm{H}), 4.90(\mathrm{dd}, J=11.0,1.2 \mathrm{~Hz}, 1 \mathrm{H}), 4.16-3.94(\mathrm{~m}, 1 \mathrm{H})$, $3.92(\mathrm{dt}, J=4.9,1.8 \mathrm{~Hz}, 1 \mathrm{H}),(2.96$ and $2.91(\mathrm{~s}, 3 \mathrm{H})), 2.72-2.49(\mathrm{~m}, 1 \mathrm{H}), 1.92-1.46(\mathrm{~m}, 3 \mathrm{H})$, 1.29-1.15 (m, 8H), (1.09 and $1.08(\mathrm{~d}, J=6.8 \mathrm{~Hz}, 3 \mathrm{H})), 0.88(\mathrm{~s}, 12 \mathrm{H}) .{ }^{13} \mathrm{C} \mathrm{NMR}(100 \mathrm{MHz}$, $\mathrm{CDCl}_{3}$ as a $1: 1$ mixture of two major conformers) $\delta 177.3$ and 176.6, 166.4, 133.4 and 133.2, $130.4,129.0,117.0$ and 116.6, 79.0, 52.1 and 50.2, 38.2, 37.1, 35.9 and 35.7, 34.9 and 34.8, 34.5 and $34.3,33.9,29.5,28.0$ and 27.9, 27.2, 26.1, 19.2, 18.2 and 17.6. HRMS (ESI) calculated for $\mathrm{C}_{22} \mathrm{H}_{39} \mathrm{NO}_{3} \mathrm{Na}^{+}[\mathrm{M}+\mathrm{Na}]^{+}$388.2822, found 388.2821.

\subsubsection{Synthesis of (2R,7S,9R)-laingolide A (1d)}

Product S21 was synthesised according to the procedures for the synthesis of S15 from 31 (30.9 mg, $85 \mu \mathrm{mol}, 1.0$ eq.). Purification of the crude product was performed using flash chromatography on silica gel (hexanes $/$ EtOAc $=5: 1$ ) to afford an inseparable mixture of the desired product $\mathbf{S 2 1}$ and a minor unidentified byproduct $(28 \mathrm{mg})$ as a white solid.

$(2 R, 7 S, 9 R)$-laingolide A (1d) was synthesised according to the procedures for the synthesis of $(2 S, 7 R, 9 R)$-laingolide A (1a) from the above mixture of the desired product S21 and a minor unidentified byproduct. Purification of the crude product was performed using flash chromatography on silica gel (hexanes/EtOAc $=5: 1)$ to afford $(2 R, 7 S, 9 R)$-laingolide A (1d) $\left(13.4 \mathrm{mg}\right.$, 47\% for two steps) as a white solid. TLC: $\mathrm{R}_{f}=0.6$ (hexanes $/$ EtOAc $=2: 1$ ), UV and PMA stain. $\alpha_{\mathrm{D}}^{23}=-55.3\left(c 0.01, \mathrm{CHCl}_{3}\right) .{ }^{1} \mathrm{H} \mathrm{NMR}\left(400 \mathrm{MHz}, \mathrm{CDCl}_{3}\right) \delta 6.74(\mathrm{~d}$, $J=13.9 \mathrm{~Hz}, 1 \mathrm{H}), 5.21(\mathrm{ddd}, J=13.8,8.6,6.3 \mathrm{~Hz}, 1 \mathrm{H}), 4.86(\mathrm{dd}, J=10.9,1.3 \mathrm{~Hz}, 1 \mathrm{H}), 3.20$ $(\mathrm{ddd}, J=16.5,6.3,1.4 \mathrm{~Hz}, 1 \mathrm{H}), 3.11(\mathrm{~s}, 3 \mathrm{H}), 3.07(\mathrm{ddd}, J=16.5,8.7,1.0 \mathrm{~Hz}, 1 \mathrm{H}), 2.68(\mathrm{dqd}$, $J=12.9,6.6,4.4 \mathrm{~Hz}, 1 \mathrm{H}), 1.63-1.50(\mathrm{~m}, 1 \mathrm{H}), 1.49-1.33(\mathrm{~m}, 3 \mathrm{H}), 1.29-1.25(\mathrm{~m}, 1 \mathrm{H}), 1.25-1.23$ $(\mathrm{m}, 2 \mathrm{H}), 1.22-1.16(\mathrm{~m}, 2 \mathrm{H}), 1.14(\mathrm{~d}, J=6.4 \mathrm{~Hz}, 4 \mathrm{H}), 1.14-1.05(\mathrm{~m}, 1 \mathrm{H}), 0.88(\mathrm{~s}, 9 \mathrm{H}), 0.85$ $(\mathrm{d}, J=5.8 \mathrm{~Hz}, 3 \mathrm{H}) .{ }^{13} \mathrm{C}$ NMR $\left(100 \mathrm{MHz}, \mathrm{CDCl}_{3}\right) \delta 176.6,171.9,132.6,106.8,80.5,38.7$, $37.5,36.2,35.6,35.0,33.7,32.2,26.9,26.6,25.3,24.9,18.9,16.6$. HRMS (ESI) calculated for $\mathrm{C}_{20} \mathrm{H}_{35} \mathrm{NO}_{3} \mathrm{Na}^{+}[\mathrm{M}+\mathrm{Na}]^{+} 360.2509$, found 360.2513 .

\section{Conclusions}

In summary, we have unambiguously established the relative and absolute configuration of laingolide A through the total synthesis of four diastereomers of the natural product. The key features of the convergent and fully stereocontrolled route included a copper-catalysed stereospecific Kumada-type coupling, a Julia-Kocienski olefination and an RCM/alkene migration sequence to access the desired macrocyclic enamide.

Supplementary Materials: The following are available online at https:/ / www.mdpi.com/article/10 $.3390 / \mathrm{md} 19050247 / \mathrm{s} 1$ : initial approaches via the cross-metathesis and ${ }^{1} \mathrm{H}$ - and ${ }^{13} \mathrm{C}-\mathrm{NMR}$ charts of all the compounds. 
Author Contributions: F.W., Y.G. and T.Y. conceived and designed this research; F.W. and T.Z. prepared the compounds and collected their spectral data; F.W., J.Y. and Y.G. analysed the experimental data; Y.G. and T.Y. prepared the manuscript. All authors have read and agreed to the published version of the manuscript.

Funding: This research was funded by the National Natural Science Foundation of China (21772009 and 21901013), the Shenzhen Peacock Plan (KQTD2015071714043444), the Shenzhen Science and Technology Innovation Commission (JCYJ20170818090017617), the GDNSF (2014B030301003) and the China Postdoctoral Science Foundation (2019M660286 for J.Y.).

Institutional Review Board Statement: Not applicable.

Informed Consent Statement: Not applicable.

Data Availability Statement: Not applicable.

Conflicts of Interest: The authors declare no conflict of interest.

\section{References}

1. Nicolaou, K.C.; Snyder, S.A. Chasing molecules that were never there: Misassigned natural products and the role of chemical synthesis in modern structure elucidation. Angew. Chem. Int. Ed. 2005, 44, 1012-1044. [CrossRef]

2. Maier, M.E. Structural revisions of natural products by total synthesis. Nat. Prod. Rep. 2009, 26, 1105-1124. [CrossRef]

3. Usami, Y. Recent synthetic studies leading to structural revisions of marine natural products. Mar. Drugs 2009, 7, 314-330. [CrossRef] [PubMed]

4. Suyama, T.L.; Gerwick, W.H.; McPhail, K.L. Survey of marine natural product structure revisions: A synergy of spectroscopy and chemical synthesis. Bioorg. Med. Chem. 2011, 19, 6675-6701. [CrossRef] [PubMed]

5. Guo, Y.; Zhao, M.; Xu, Z.; Ye, T. Total synthesis and stereochemical assignment of actinoranone. Chem. Eur. J. 2017, 23, 3572-3576. [CrossRef]

6. Zhou, J.; Gao, B.; Xu, Z.; Ye, T. Total synthesis and stereochemical assignment of callyspongiolide. J. Am. Chem. Soc. 2016, 138, 6948-6951. [CrossRef] [PubMed]

7. Liu, J.; Wang, L.; Zhang, J.; Xu, Z.; Ye, T. The total synthesis and stereochemical assignment of scytonemin A. Chem. Commun. 2016, 52, 1002-1005. [CrossRef]

8. Qu, S.; Chen, Y.; Wang, X.; Chen, S.; Xu, Z.; Ye, T. Total synthesis of largamide B. Chem. Comm. 2015, 51, 2510-2513. [CrossRef]

9. Lei, H.; Yan, J.; Yu, J.; Liu, Y.; Wang, Z.; Xu, Z.; Ye, T. Total synthesis and stereochemical reassignment of mandelalide A. Angew. Chem. Int. Ed. 2014, 53, 6533-6537. [CrossRef]

10. Dai, L.; Chen, B.; Lei, H.; Wang, Z.; Liu, Y.; Xu, Z.; Ye, T. Total synthesis and stereochemical revision of lagunamide A. Chem. Commun. 2012, 48, 8697-8699. [CrossRef]

11. Gao, X.; Liu, Y.; Kwong, S.; Xu, Z.; Ye, T. Total synthesis and stereochemical reassignment of bisebromoamide. Org. Lett. 2010, 12, 3018-3021. [CrossRef]

12. Klein, D.; Braekman, J.C.; Daloze, D.; Hoffmann, L.; Castillo, G.; Demoulin, V.V. Madangolide and laingolide A, two novel macrolides from Lyngbya bouillonii (Cyanobacteria). J. Nat. Prod. 1999, 62, 934-936. [CrossRef]

13. Klein, D.; Braekman, J.C.; Daloze, D.; Hoffmann, L.; Demoulin, V. Laingolide, a novel 15-membered macrolide from Lyngbya bouillonii (cyanophyceae). Tetrahedron Lett. 1996, 37, 7519-7520. [CrossRef]

14. Matthew, S.; Salvador, L.A.; Schupp, P.J.; Paul, V.J.; Luesch, H. Cytotoxic halogenated macrolides and modified peptides from the apratoxin-producing marine cyanobacterium Lyngbya bouillonii from Guam. J. Nat. Prod. 2010, 73, 1544-1552. [CrossRef] [PubMed]

15. Pereira, A.R.; Cao, Z.; Engene, N.; Soria-Mercado, I.E.; Murray, T.F.; Gerwick, W.H. Palmyrolide A, an unusually stabilized neuroactive macrolide from Palmyra Atoll cyanobacteria. Org. Lett. 2010, 12, 4490-4493. [CrossRef] [PubMed]

16. Yadav, J.S.; Suresh, B.; Srihari, P. Expedient synthesis of large-ring trans-enamide macrolides by CuI-mediated intramolecular coupling of vinyl iodide with amide: Total synthesis of palmyrolide A. Eur. J. Org. Chem. 2016, 2016, 2509-2513. [CrossRef]

17. Philkhana, S.C.; Mehrotra, S.; Murray, T.F.; Reddy, D.S. Synthesis and biological evaluation of palmyrolide A macrocycles as sodium channel blockers towards neuroprotection. Org. Bio. Chem. 2016, 14, 8457-8473. [CrossRef] [PubMed]

18. Borra, S.; Amrutapu, S.K.; Pabbaraja, S.; Singh, Y.J. Stereoselective total synthesis of palmyrolide A via intramolecular trans N-methyl enamide formation. Tetrahedron Lett. 2016, 57, 4456-4459. [CrossRef]

19. Wadsworth, A.D.; Furkert, D.P.; Brimble, M.A. Total synthesis of the macrocyclic N-Methyl enamides palmyrolide A and 2S-sanctolide A. J. Org. Chem. 2014, 79, 11179-11193. [CrossRef]

20. Sudhakar, G.; Reddy, K.J.; Nanubolu, J.B. Total synthesis of palmyrolide A and its 5,7-epi isomers. Tetrahedron 2013, 69, 2419-2429. [CrossRef]

21. Philkhana, S.C.; Seetharamsingh, B.; Dangat, Y.B.; Vanka, K.; Reddy, D.S. Synthesis of palmyrolide A and its cis-isomer and mechanistic insight into trans-cis isomerisation of the enamide macrocycle. Chem. Comm. 2013, 49, 3342-3344. [CrossRef] 
22. Wadsworth, A.D.; Furkert, D.P.; Sperry, J.; Brimble, M.A. Total synthesis of the initially reported and revised structures of the neuroprotective agent palmyrolide A. Org. Lett. 2012, 14, 5374-5377. [CrossRef] [PubMed]

23. Tello-Aburto, R.; Newar, T.D.; Maio, W.A. Evolution of a protecting-group-free total synthesis: Studies en route to the neuroactive marine macrolide(-)-palmyrolide A. J. Org. Chem. 2012, 77, 6271-6289. [CrossRef]

24. Tello-Aburto, R.; Johnson, E.M.; Valdez, C.K.; Maio, W.A. Asymmetric total synthesis and absolute stereochemistry of the neuroactive marine macrolide palmyrolide A. Org. Lett. 2012, 14, 2150-2153. [CrossRef]

25. Phansavath, P.; Pomey, G. Total synthesis of laingolide A diastereomers. Synthesis 2015, 47, 1016-1023. [CrossRef]

26. Nitelet, A.; Jouvin, K.; Evano, G. Development of a general copper-catalyzed vinylic Finkelstein reaction-application to the synthesis of the C1-C9 fragment of laingolide B. Tetrahedron 2016, 72, 5972-5987. [CrossRef]

27. Cui, C.; Dai, W.M. Total synthesis of laingolide B stereoisomers and assignment of absolute configuration. Org. Lett. 2018, 20, 3358-3361. [CrossRef]

28. Cui, C.; Lai, Y.; Dai, W.M. Multimodule Assembly Strategy for Diverted Total Synthesis and Stereochemical Determination of Laingolide A and Laingolide. ChemRxiv 2021. Available online: https:/ / chemrxiv.org/articles/preprint/Multimodule_Assembly_ Strategy_for_Diverted_Total_Synthesis_and_Stereochemical_Determination_of_Laingolide_A_and_Laingolide/14347145 (accessed on 7 April 2021). (While Our Work Was in Progress, a Publication by Dai and co-Workers Appeared Describing Asymmetric Synthesis of (-)-laingolide A.).

29. Schwab, P.; Grubbs, R.H.; Ziller, J.W. Synthesis and application of $\mathrm{RuCl}_{2}\left(=\mathrm{CHR}^{\prime}\right)\left(\mathrm{PR}_{3}\right)_{2}$ : The influence of the alkylidene moiety on metathesis activity. J. Am. Chem. Soc. 1996, 118, 100-110. [CrossRef]

30. Schwab, P.; France, M.B.; Ziller, J.W.; Grubbs, R.H. A series of well-defined metathesis catalysts-synthesis of $\left[\mathrm{RuCl}_{2}\left(\mathrm{CHR}^{\prime}\right)\left(\mathrm{PR}_{3}\right)_{2}\right]$ and its reactions. Angew. Chem. Int. Ed. 1995, 34, 2039-2041. [CrossRef]

31. Kingsbury, J.S.; Harrity, J.P.A.; Bonitatebus, P.J.; Hoveyda, A.H. A recyclable Ru-based metathesis catalyst. J. Am. Chem. Soc. 1999, 121, 791-799. [CrossRef]

32. Scholl, M.; Ding, S.; Lee, C.W.; Grubbs, R.H. Synthesis and activity of a new generation of ruthenium-based olefin metathesis catalysts coordinated with 1,3-dimesityl-4,5-dihydroimidazol-2-ylidene ligands. Org. Lett. 1999, 1, 953-956. [CrossRef]

33. Garber, S.B.; Kingsbury, J.S.; Gray, B.L.; Hoveyda, A.H. Efficient and recyclable monomeric and dendritic Ru-based metathesis catalysts. J. Am. Chem. Soc. 2000, 122, 8168-8179. [CrossRef]

34. Chatterjee, A.K.; Choi, T.L.; Sanders, D.P.; Grubbs, R.H. A general model for selectivity in olefin cross metathesis. J. Am. Chem. Soc. 2003, 125, 11360-11370. [CrossRef] [PubMed]

35. Fürstner, A.; Langemann, K. Total syntheses of (+)-ricinelaidic acid lactone and of (-)-gloeosporone based on transition-metalcatalyzed C-C bond formations. J. Am. Chem. Soc. 1997, 119, 9130-9136. [CrossRef]

36. Abell, A.D.; Alexander, N.A.; Aitken, S.G.; Chen, H.; Coxon, J.M.; Jones, M.A.; McNabb, S.B.; Muscroft-Taylor, A. Synthesis of macrocyclic beta-strand templates by ring closing metathesis. J. Org. Chem. 2009, 74, 4354-4356. [CrossRef]

37. Ramirez-Contreras, R.; Morandi, B. Chemo- and regioselective functionalization of polyols through catalytic $\mathrm{C}\left(\mathrm{sp}^{3}\right)-\mathrm{C}\left(\mathrm{sp}^{3}\right)$ Kumada-type coupling of cyclic sulfate esters. Org. Lett. 2016, 18, 3718-3721. [CrossRef]

38. Baudin, J.B.; Hareau, G.; Julia, S.A.; Ruel, O. A direct synthesis of olefins by reaction of carbonyl compounds with lithio derivatives of 2-[alkyl- or (2'-alkenyl)- or benzyl-sulfonyl]-benzothiazoles. Tetrahedron Lett. 1991, 32, 1175-1178. [CrossRef]

39. Blakemore, P.R.; Cole, W.J.; Kocienski, P.J.; Morley, A. A stereoselective synthesis of trans-1,2-disubstituted alkenes based on the condensation of aldehydes with metallated 1-phenyl-1H-tetrazol-5-yl sulfones. Synlett 1998, 26-28. [CrossRef]

40. Blakemore, P.R. The modified Julia olefination: Alkene synthesis via the condensation of metallated heteroarylalkylsulfones with carbonyl compounds. J. Chem. Soc. Perkin Trans. 1 2002, 2563-2585. [CrossRef]

41. List, B.; Lerner, R.A.; Barbas, C.F. Proline-catalyzed direct asymmetric aldol reactions. J. Am. Chem. Soc. 2000, 122, 2395-2396. [CrossRef]

42. Gilles, A.; Martinez, J.; Cavelier, F. Supported synthesis of oxoapratoxin A. J. Org. Chem. 2009, 74, 4298-4304. [CrossRef] [PubMed]

43. Lucio Anelli, P.; Biffi, C.; Montanari, F.; Quici, S. Fast and selective oxidation of primary alcohols to aldehydes or to carboxylic acids and of secondary alcohols to ketones mediated by oxoammonium salts under two-phase conditions. J. Org. Chem. 1987, 52, 2559-2562. [CrossRef]

44. Evans, D.A.; Chapman, K.T.; Carreira, E.M. Directed reduction of $\gamma$-hydroxy ketones employing tetramethyl-ammonium triacetoxyborohydride. J. Am. Chem. Soc. 1988, 110, 3560-3578. [CrossRef]

45. Li, B.; Masuda, S.; Minato, D.; Zhou, D.; Sugimoto, K.; Nemoto, H.; Matsuya, Y. Stereoselective approach to potential scaffold of A-nor B-aromatic OSW-1 analogues via [4+2] cycloaddition of o-quinodimethane. Tetrahedron 2014, 70, 3981-3987. [CrossRef]

46. Yang, M.; Peng, W.; Guo, Y.; Ye, T. Total synthesis of dysoxylactam A. Org. Lett. 2020, 22, 1776-1779. [CrossRef]

47. Epp, J.B.; Widlanski, T.S. Facile preparation of nucleoside-5'-carboxylic acids. J. Org. Chem. 1999, 64, 293-295. [CrossRef]

48. Yue, C.J.; Liu, Y.; He, R. Olefins isomerization by hydride-complexes of ruthenium. J. Mol. Catal. A Chem. 2006, 259, 17-23. [CrossRef] 